\title{
The Role of Preparation in Tuning Anticipatory and Reflex Responses During Catching
}

\author{
Francesco Lacquaniti and Claudio Maioli \\ Istituto di Fisiologia dei Centri Nervosi, CNR, Milan, Italy
}

The pattern of muscle responses associated with catching a ball in the presence of vision was investigated by independently varying the height of the drop and the mass of the ball. It was found that the anticipatory EMG responses comprised early and late components. The early components were produced at a roughly constant latency (about $130 \mathrm{msec}$ ) from the time of ball release. Their mean amplitude decreased with increasing height of fall. Late components represented the major build-up of muscle activity preceding the ball's impact and were accompanied by limb flexion. Their onset time was roughly constant (about 100 msec) with respect to the time of impact (except in wrist extensors). This indicates that the timing of these responses was based on an accurate estimate of the instantaneous values of the time-to-contact (time remaining before impact). The mean amplitude of the late anticipatory responses increased linearly with the expected momentum of the ball at impact. The reflex responses evoked by the ball's impact consisted in a short-latency coactivation of flexor and extensor muscles at the elbow and wrist joints. Their mean amplitude generally increased with the intensity of the perturbation both in the stretched muscles and in the shortening muscles. We argue that both the anticipatory and the reflex coactivation are centrally preset in preparation for catching and are instrumental for stabilizing limb posture after impact. A model with linear, time-varying viscoelastic coefficients was used to assess the neural and mechanical contributions to the damping of limb oscillations induced by the ball's impact. The model demonstrates that (1) anticipatory muscle stiffening and anticipatory flexion of the limb are synergistic in building up resistance of the hand to vertical displacement and (2) the reflex coactivation produces a further increment of hand stiffness and viscosity which tends to offset the decrement which would result from the limb extension produced by the impact.

The role of preparation in tuning motor behavior according to the sensory information and the cognitive representation about a specific task has long attracted considerable interest (e.g., Evarts et al., 1984; Kornblum and Requin, 1984; Georgopoulos and Massey, 1988). In particular, reaction-time paradigms have been utilized to demonstrate that providing advance information about the timing, amplitude, or direction of a command signal can

Received Feb. 10, 1988; revised May 17, 1988; accepted May 23, 1988.

We wish to thank Dr. Carlo Terzuolo for a critical reading of the manuscript.

Correspondence should be addressed to Dr. Francesco Lacquaniti, Istituto di Fisiologia dei Centri Nervosi, CNR, Via Mario Bianco 9, 20131 Milan, Italy.

Copyright $(C) 1989$ Society for Neuroscience $0270-6474 / 89 / 010134-15 \$ 02.00 / 0$ increase the speed and accuracy of the response (see Georgopoulos et al., 1981; Gordon and Ghez, 1987). Also the reflex behavior has been shown to be set-related (Hammond et al., 1956; Melvill Jones and Watt, 1971; Nashner, 1977); for instance, the amplitude of myotatic responses elicited by means of torque motor perturbations depends on whether or not such perturbations are expected, as well as on the particular task being executed (Gottlieb and Agarwal, 1980; Soechting et al., 1981; Akazawa el al., 1983).

However, most studies on preparation have concentrated on rather simple motor tasks, involving single-joint motion. The limitations inherent in such an approach have recently become apparent with the recognition that the organizing principles of limb coordination and the modalities of operation of limb reflexes in unconstrained behavior cannot be readily derived from more restricted observations (Nashner, 1977; Traub et al., 1980; Abbs and Gracco, 1984; Lacquaniti and Soechting, 1984; Cole and Abbs, 1987).

Catching a moving ball is a natural paradigm for the study of motor preparation, yet it has received scant attention (Alderson et al., 1974; Sharp and Whiting, 1975; Lee, 1980). This task involves the coordinated action of several limb muscles acting on different limb joints within rigid spatiotemporal constraints. Successful performance is, in fact, predicated upon the ability to intercept the ball trajectory within a narrow time span. Accordingly, the sequence of kinematic events that lead to the correct orientation of the limb in space prior to contact shows little time variability (Alderson et al., 1974). Not only the trajectory but also the compliance of the limb needs to be accurately controlled during catching. The latter parameter, in particular, plays a crucial role in the dynamic interaction with the ball. Both the absorption of momentum and the subsequent grasping of the ball rely on an appropriate matching of the limb compliance with the properties of the impulsive impact. For instance, if the compliance is too high, the hand will yield; if it is too low, the ball will rebound.

The factors that enter into the adjustment of limb compliance before and after impact are poorly understood. One would expect that both visual information and cognitive inferences about the ball's motion are used to predict impact parameters (e.g., timc, forcc, clasticity) and to adjust compliancc accordingly. In principle, the control of compliance could be achieved by dispatching appropriate levels of muscle activation prior to impact (see Humphrey and Reed, 1983) and by presetting reflex and reaction-time responses evoked by the impact (see Houk and Rymer, 1981). For instance, it is known that bursts of activity in limb muscles, either triggered by sensory stimuli or centrally programmed, precede and accompany landing from a fall or jump (Melvill Jones and Watt, 1971; Dietz and Noth, 1978; 
McKinley and Smith, 1983). In this case, anticipatory activity serves to stiffen the limbs in preparation for the impact with the ground. In contrast, functional stretch reflexes elicited by landing are inappropriately timed to stabilize posture, due to the long neural delays involved, and can be actively suppressed.

In a preliminary note (Lacquaniti and Maioli, 1987), we have begun to address the problem of how limb compliance is controlled in a task that involves catching a free-falling ball. It was found that flexor and extensor muscles of wrist and elbow joints are coactivated not only in anticipation of the impact of the ball, but also at a short latency following impact. By deceiving the subjects on the exact timing of impact, we were able to demonstrate the reflex nature of the short-latency EMG responses following the impact. ${ }^{1}$ Thus, the classical principle of reciprocal innervation, traditionally deemed to apply to stretch reflexes (see Matthews, 1972), is violated by the reflex responses evoked by ball impact inasmuch as stretched and shortening muscles are both activated by the stimulus.

In this paper we describe in more detail both the anticipatory and reflex behaviors associated with catching in the presence of vision. Several questions raised by the previous study will be addressed. First, the question of which parameters of ball motion the anticipatory and reflex actions are geared to is addressed by experimentally manipulating the value of the height of fall and the mass of the ball. Changes in the height of fall affect both the time and magnitude of ball impact: the higher the drop, the greater the duration of fall, velocity, and momentum at impact. On the other hand, changes in ball mass for a given height affect only the momentum at impact. Thus, the status of these spatiotemporal variables as the potential sensory-perceptual determinants of motor actions can be assessed vis-à-vis changes in the timing and amplitude of anticipatory and reflex responses.

A second question we address here is whether the observed pattern of coactivation of flexor and extensor muscles of wrist and elbow joints can result in a time-varying modulation of the compliance at the hand which is appropriate to the dynamic interaction with the falling ball. In a companion paper, we deal with the problem of the adaptation of catching behavior in the absence of visual information.

\section{Materials and Methods}

The general experimental procedures have been described previously (Lacquaniti and Maioli, 1987). Briefly, subjects were asked to catch balls ( $9 \mathrm{~cm}$ in diameter) dropped by an electromagnet from various heights $(0.2-1.2 \mathrm{~m})$ at random times $(1-4.5 \mathrm{sec})$ after a warning tone (Fig. 1). They were seated with their right arm strapped to a goniometer, which measured the angle of flexion-extension at the elbow $(\Theta)$ and wrist $(\Phi)$. The elbow joint was aligned with the shaft of a low-inertia torque motor, which constantly applied a torque equal and opposite to the gravitational torque on the goniometer. The hand was fully supinated, and subjects wore a stiff glove. The horizontal position of the electromagnet was adjusted so that, with the forearm and hand horizontal, the metacarpophalangeal joint of the middle finger was on the ball's line of fall.

The EMG activities of biceps, triceps, flexor carpi radialis (FCR) flexor carpi ulnaris (FCU), extensor carpi radialis (ECR), and extensor carpi ulnaris (ECU) were recorded by means of surface electrodes (SensorMedics 650414; diameter of the detection surface, $2 \mathrm{~mm}$ ) in bipolar configuration. Interdetection spacing was about $1-2 \mathrm{~cm}$ for best selectivity. Electrode placement was always carefully chosen so as to minimize cross-talk from adjacent muscles during maximal isometric contractions.

Experimental protocol. Ten male subjects between 25 and 59 years of age volunteered for the experiments. They were instructed to position

${ }^{1}$ Note added in proof: More recently, these findings have also been reported by a different group under similar experimental conditions (Johansson and Westling, 1988).

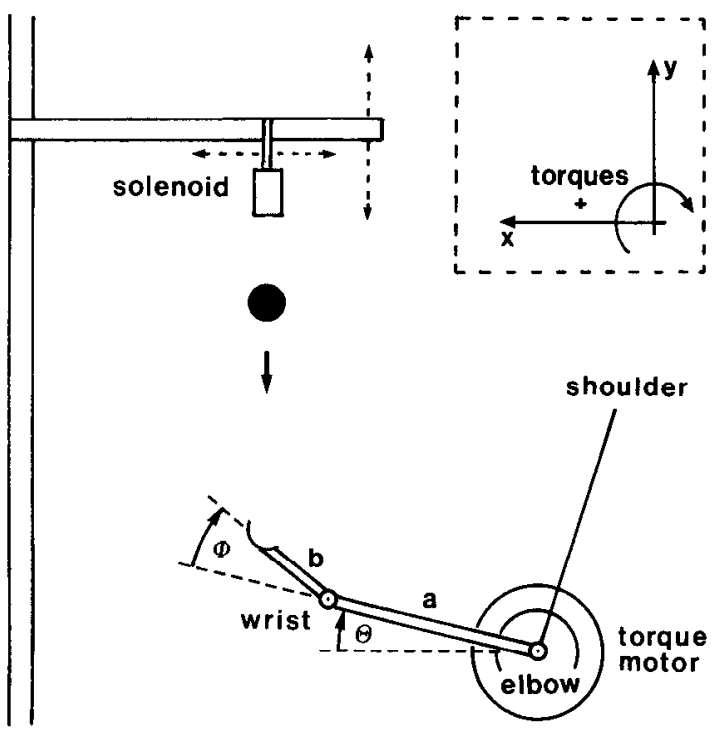

Figure 1. Schematic of the experimental set-up. Hand and forearm were strapped to a 2-degrees-of-freedom electrogoniometer which measured elbow $(\Theta)$ and wrist $(\Phi)$ angles. Elbow joint was aligned with the shaft of a torque motor which constantly applied a torque equal to and opposite that of the gravitational torque on the goniometer. The hand was fully supinated, and subjects wore a stiff glove. The ball was dropped by an electromagnet from variable heights. The horizontal position of the electromagnet was adjusted so that, with the forearm and hand horizontal, the metacarpophalangeal joint of the middle finger was on the line of fall of the ball.

their hand on the line of ball's fall, get ready at the warning, and catch and hold the ball through the duration of the trial $(1.5 \mathrm{sec}$ from ball release). Vision was permitted throughout. Although no specific instructions were given with regard to the direction of gaze, subjects generally fixated the ball prior to release. No practice trials were allowed.

In order to investigate the repertoire of motor behaviors associated with scalar changes in the parameters of ball impact, the experiments followed a $3 \times 3$ factorial design: 3 balls of different mass but identical size and appearance were dropped from 3 different heights. Thus, all experiments comprised 9 different series with blocks of 13 consecutive trials each, the order of presentation being randomized across experiments. By varying the height of fall $(h)$, the theoretical duration of fall $(d=\sqrt{ } 2 h / g)$, as well as the velocity $(v=\sqrt{ } 2 h g)$ and momentum $(m v)$ of the ball at impact were changed. By varying ball mass $(m)$ for any given $h$, instead, only the momentum was changed. In an initial group of 7 experiments (experiment $A$ in Table 1), the values of $m$ and $h$ were chosen so that the values of $m v$ were about equal $(6 \%$ of maximum deviation) along the diagonals. In a second group of 4 experiments (experiment $\mathrm{B}$ in Table 1), the upper range of $h$ investigated was extended beyond that of experiment $\mathrm{A}$, and the 9 values of $m v$ were almost equispaced.

To avoid fatigue during the experiments, limb weight was supported between trials, and rest was allowed between blocks of trials.

Data analysis. Kincmatic and EMG data were sampled on line by a computer at $1 \mathrm{kHz}$, starting from $128 \mathrm{msec}$ before ball release. The EMG activities were rectified off line. The kinematic data were numerically filtered by means of an optimal low-pass FIR (finite impulse response) filter (27-points, cutoff at $0.18 \times$ sampling frequency) and differentiated (16-points optimal FIR differentiator; Rabiner and Gold, 1975). All trials, except the first 3, of each series were ensemble-averaged using as temporal reference the instant of impact of the ball with the hand. This was estimated as the time at which wrist velocity crossed a fixed threshold. The torques acting at the elbow $\left(T_{c}\right)$ and wrist $\left(T_{w}\right)$ were computed according to classical mechanics using the differentiated kinematic data (Soechting and Lacquaniti, 1981):

$$
\begin{aligned}
T_{c}= & \left(I_{c}+I_{n}+2 A \cos \phi\right) \ddot{\Theta}+\left(I_{w}+A \cos \Phi\right) \ddot{\Phi}-A \sin \Phi\left(\dot{\Phi}^{2}+2 \dot{\Phi} \dot{\Theta}\right) \\
& +B \cos \Theta+C \cos (\Theta+\Phi), \\
T_{w}= & \left(I_{w}+A \cos \Phi\right) \ddot{\Theta}+I_{w} \ddot{\Phi}+A \sin \Phi \dot{\Theta}^{2}+C \cos (\Theta+\Phi) .
\end{aligned}
$$


Table 1. Experimental protocols

\begin{tabular}{lllll} 
& & \multicolumn{3}{c}{$\begin{array}{l}\text { Momentum }(\mathrm{kg} \mathrm{m} / \mathrm{sec}) \text { at } \\
\text { indicated } h(\mathrm{~m})\end{array}$} \\
\cline { 3 - 5 } & $\begin{array}{llll}m \\
(\mathrm{~kg})\end{array}$ & 0.2 & 0.4 & 0.8 \\
\hline Experiment $\mathrm{A}$ & 0.8 & 1.58 & 2.24 & 3.17 \\
& 0.6 & 1.19 & 1.68 & 2.38 \\
& 0.4 & 0.79 & 1.12 & 1.58
\end{tabular}

Momentum $(\mathrm{kg} \mathrm{m} / \mathrm{sec})$ at

\begin{tabular}{|c|c|c|c|c|}
\hline & \multirow{2}{*}{$\begin{array}{l}m \\
(\mathrm{~kg})\end{array}$} & \multicolumn{3}{|c|}{ indicated $h(\mathrm{~m})$} \\
\hline & & 0.4 & 0.8 & 1.2 \\
\hline \multirow[t]{3}{*}{ Experiment B } & 0.6 & 1.68 & 2.38 & 2.91 \\
\hline & 0.4 & 1.12 & 1.58 & 1.94 \\
\hline & 0.2 & 0.56 & 0.79 & 0.97 \\
\hline
\end{tabular}

The duration of fall $(d)$ is $202,286,404$, and $495 \mathrm{msec}$ for height of fall $(h)$ of $0.2,0.4,0.8$, and $1.2 \mathrm{~m}$, respectively.

$I_{s}$ and $I_{w}$ are the moments of inertia of the forearm and hand about elbow and wrist, respectively. These coefficients as well as $A, B$, and $C$ are constants (for details, see Soechting and Lacquaniti, 1981). Their values were computed on the basis of anthropometric data for each subject. (After impact time, the mass of the ball was added to that of the hand.) As computed, $T_{e}$ and $T_{w}$ correspond to the net nonconservative torques in the flexor direction at each joint. They result from the external force applied to the limb at ball impact and from internal forces generated by the contractile and viscoelastic properties of muscles.

Anticipatory EMG responses comprised early and late components. They were quantitatively analyzed in the following way. After smoothing the EMG (50 Hz cutoff), the mean and $95 \%$ confidence limits were computed over the $128 \mathrm{msec}$ interval preceding ball release and subtracted. The latency of the early anticipatory responses was estimated as the time at which EMG first exceeded and remained above the baseline confidence limits for more than 10 consecutive samples. By contrast, the onset of the late anticipatory responses was defined as the time at which, proceeding in the backward direction along the time axis from impact to start, the EMG first decreased below $25 \%$ of the maximum value reached before impact. Their mcan amplitude was cvaluated over the $50 \mathrm{msec}$ interval preceding ball impact.

The EMG responses to impact were, instead, analyzed as follows. EMG activity (unsmoothed) was averaged over the $20 \mathrm{msec}$ centered on impact time (prereflex baseline). The latency of the responses was defined as the time at which, starting from impact, the EMG first exceeded the $95 \%$ confidence limits of this baseline. Their mean amplitude was calculated over the $15-40 \mathrm{msec}$ interval following impact, and the prereflex baseline was subtracted (see Gottlieb and Agarwal, 1980; Soechting et al., 1981)

\section{Results}

The salient results are exemplified in Figure 2 using the raw data of a single trial. About $100 \mathrm{msec}$ before impact (indicated by the vertical line), EMG activity built up in the flexor and extensor muscles at the elbow and wrist joints. Such anticipatory activity was associated with wrist flexion. The momentum of the ball resulted in an impulsive perturbation that extended wrist and elbow joints. This perturbation evoked short-latency $(15-20 \mathrm{msec})$ reflex coactivation of flexor and extensor muscles of bricf duration. In the following, we shall first consider the anticipatory behavior and subsequently the reflex behavior.

\section{Time course of the anticipatory responses}

The effect of varying the height of fall and mass of the ball on the anticipatory responses was investigated in experiments such as those illustrated in Figure 3. Ensemble averages from $2 \mathrm{ex}$ periments ( 2 subjects) are plotted in panels $A-C$ and $D-F$, respectively.

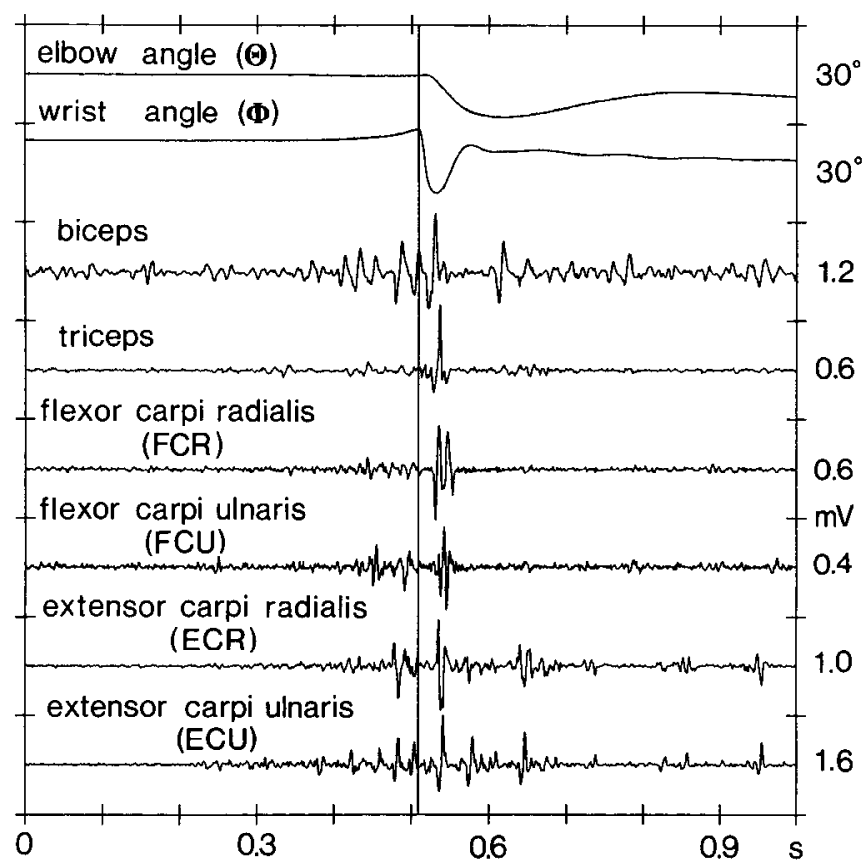

Figure 2. Raw data of a single trial from an experiment in which a $0.4 \mathrm{~kg}$ ball was dropped from $1.2 \mathrm{~m}$ in about $500 \mathrm{msec}$. The vertical line denotes the time of impact. Traces from top to bottom correspond to elbow angle $(\Theta)$, wrist angle $(\Phi)$, EMG activity of biceps, triceps, FCR, FCU, ECR, and ECU.

All subjects consistently elected an initial position of the forearm slightly flexed relative to the horizontal $\left(\Theta=12^{\circ} \pm 3^{\circ}\right.$, mean \pm SD over 99 experimental series with 10 subjects) with the hand slightly extended $\left(\Phi=-8^{\circ} \pm 3^{\circ}\right)$. While they did not move their limb prior to impact during drops from $0.2 \mathrm{~m}$ (Fig. $3 A$ ), a small but highly consistent flexion occurred within the final $100 \mathrm{msec}$ of higher drops (Fig. 3, $B-F$ ). Wrist flexion began 72 $\pm 42 \mathrm{msec}(n=78)$ before impact and reached a maximum of $5^{\circ} \perp 3^{\circ}$ at impact. Elbow flexion began $60 \pm 34 \mathrm{msec}$ prior to impact and reached a maximum of $4^{\circ} \pm 2^{\circ}$.

Drops from $0.2 \mathrm{~m}$ were followed by a single anticipatory EMG response at a latency of 120-140 msec. Higher drops, in contrast, were associated with multiphasic anticipatory responses. An early burst of EMG activity with a latency of $120-140 \mathrm{msec}$ and a duration of 30-50 msec can be detected in the EMG of the elbow muscles in Figure $3, B-F$, but only in Figure $3 B$ for wrist muscles. In general, a statistically significant early response was present in $70 \%$ of the experiments with $h>0.2 \mathrm{~m}$ for biceps, and in $35-60 \%$ for the other muscles.

Late responses, however, were always present following drops greater than $0.2 \mathrm{~m}$. They consisted in a gradual but substantial increment of activity whose latency increased with height. Thus, the onset of the late responses in biceps, triceps, and FCR occurred around $170 \mathrm{msec}$ after ball release in Figure $3 B(h=0.4$ $\mathrm{m})$, at about $300 \mathrm{msec}$ in Figurc $3, C, D(h=0.8 \mathrm{~m})$, and 400 msec in Figure 3, $E, F(h=1.2 \mathrm{~m})$. As a result, the duration of this activity (about $100 \mathrm{msec}$ ) varied little from case to case. By contrast, the duration of ECR responses increased appreciably with height (see below). None of the latencies of the muscle responses changed appreciably with the specific mass of the ball dropped from a given height (cf. Fig. 3,E, F). Often, the EMG responses preceding impact did not increase monotonically but tended to subside just before impact time (cf. biceps and triceps 

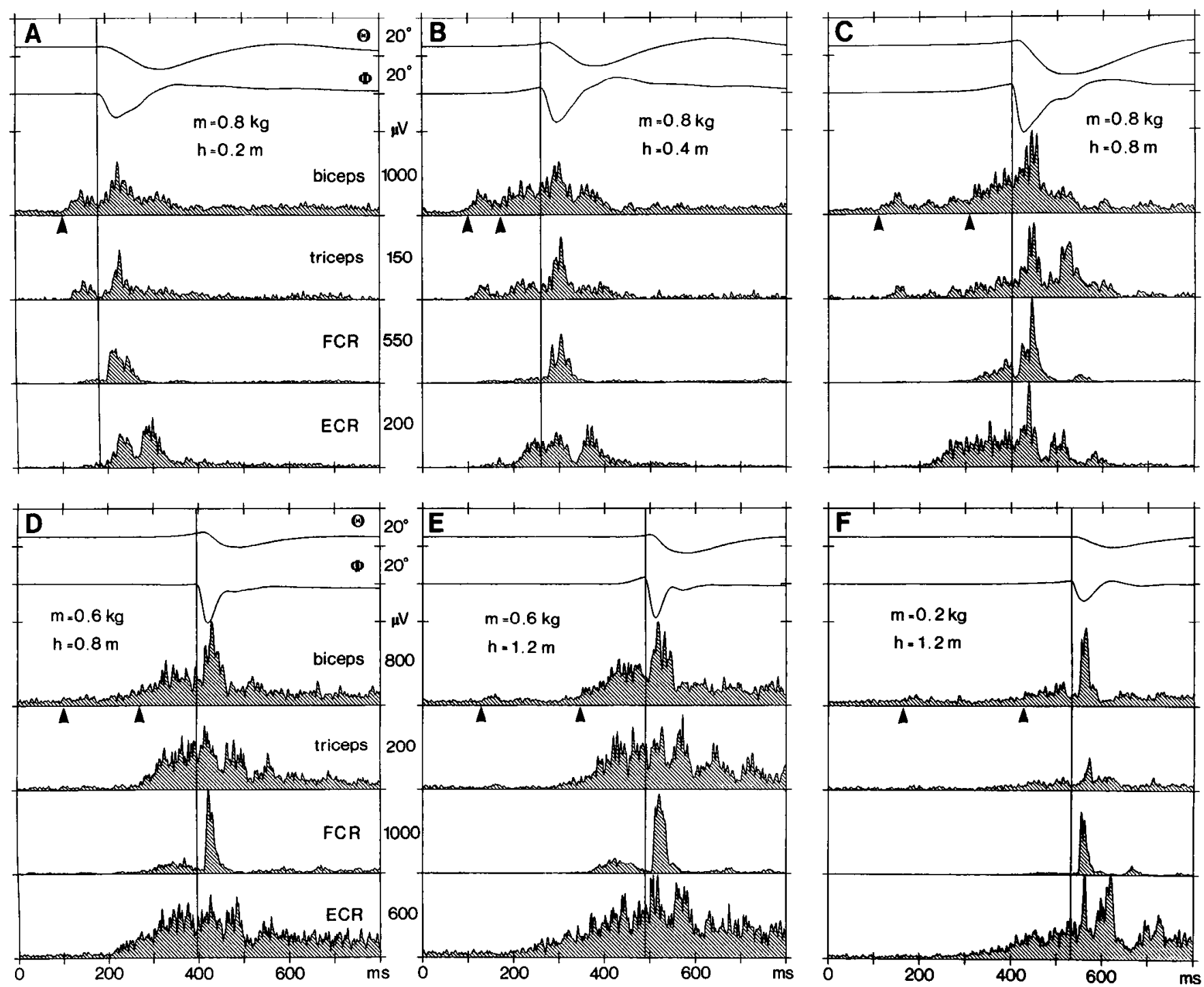

Figure 3. Ensemble averages from 2 experiments ( 2 subjects) are plotted in $A-C$ and $D-F$, respectively. The mass of the ball and the height of fall are indicated in each panel. The vertical lines denote the time of impact. Traces from top to bottom correspond to elbow angle $(\theta)$, wrist angle $(\Phi)$, rectified EMG activity of biceps, triceps, FCR, and ECR. The indicated scales apply to all panels of each experiment. The computed times of onset of the early and late anticipatory responses of biceps are indicated by the arrows.

in Fig. 3, $A, E, F$, and FCR in Fig. 3, $C, E$ ). On average, the maximum anticipatory activity occurred at $28 \pm 9 \mathrm{msec}$ before impact in biceps, $42 \pm 16 \mathrm{msec}$ in triceps, $22 \pm 11 \mathrm{msec}$ in FCR, and $23 \pm 12 \mathrm{msec}$ in ECR.

Data reduction in the format of Figure 4 allows a general assessment of the relationship between the time course of EMG anticipatory responses and height of fall. The traces in each panel correspond to the grand averages $( \pm 1 \mathrm{SD})$ of the results obtained in all experiments at the indicated heights. For averaging purposes, individual EMG responses have been scaled to the maximum reached prior to impact (after subtraction of the baseline) and aligned relative to impact time. Each sample is characterized by a limited variability in the time course of EMG activities. The grand averages of wrist muscles activities exhibit a single anticipatory response. (The time courses of FCU and ECU were very similar to those of their synergists, FCR and ECR, respectively.) By contrast, distinct early and late components are apparent in the average anticipatory responses of elbow muscles to drops greater than $0.4 \mathrm{~m}$ (except for triceps at $h=1.2 \mathrm{~m}$ ), but they tend to merge during lower drops. While early components were roughly time-locked to the release of the ball, late components were roughly time-locked to the impact.

The latency of the early components was computed in individual ensemble averages (see Materials and Methods). The mean values over 21 experimental series involving drops from $0.2 \mathrm{~m}$ were as follows: $124 \pm 14 \mathrm{msec}$ for biceps, $129 \pm 48$ msec for triceps, $136 \pm 49 \mathrm{msec}$ for FCR, $125 \pm 41 \mathrm{msec}$ for ECR. In biceps, the mean latency was $125 \pm 41 \mathrm{msec}$ for drops from $0.4 \mathrm{~m}, 132 \pm 21 \mathrm{msec}$ from $0.8 \mathrm{~m}$, and $137 \pm 31 \mathrm{msec}$ from $1.2 \mathrm{~m}$.

The time of onset of the late responses was computed relative to the time of impact. The mean values (and SD) over all experiments and subjects are reported in Table 2. The increase of the mean values with height, although statistically significant $[F(3,9)=8.3, p<0.01$, in a 2-way analysis of variance, $4 \mathrm{EMG}$ $\times 4$ heights], was limited. Moreover, most of the variance is accounted for by (1) the transition between $h=0.2 \mathrm{~m}$ and $h=$ $0.4 \mathrm{~m}$, and (2) the behavior of ECR. In ECR, in fact, the duration 
Figure 4. General time course of EMG anticipatory responses. The traces in each panel correspond to the grand averages $( \pm 1 \mathrm{SD})$ of the results obtained in all experiments at the indicated heights $(n=21,33,33,12$ for $h=0.2$, $0.4,0.8,1.2 \mathrm{~m}$, respectively). For averaging purposes, individual EMG responses have been scaled to the maximum reached prior to impact (after subtraction of the baseline) and aligned relative to impact time $(t=0)$.
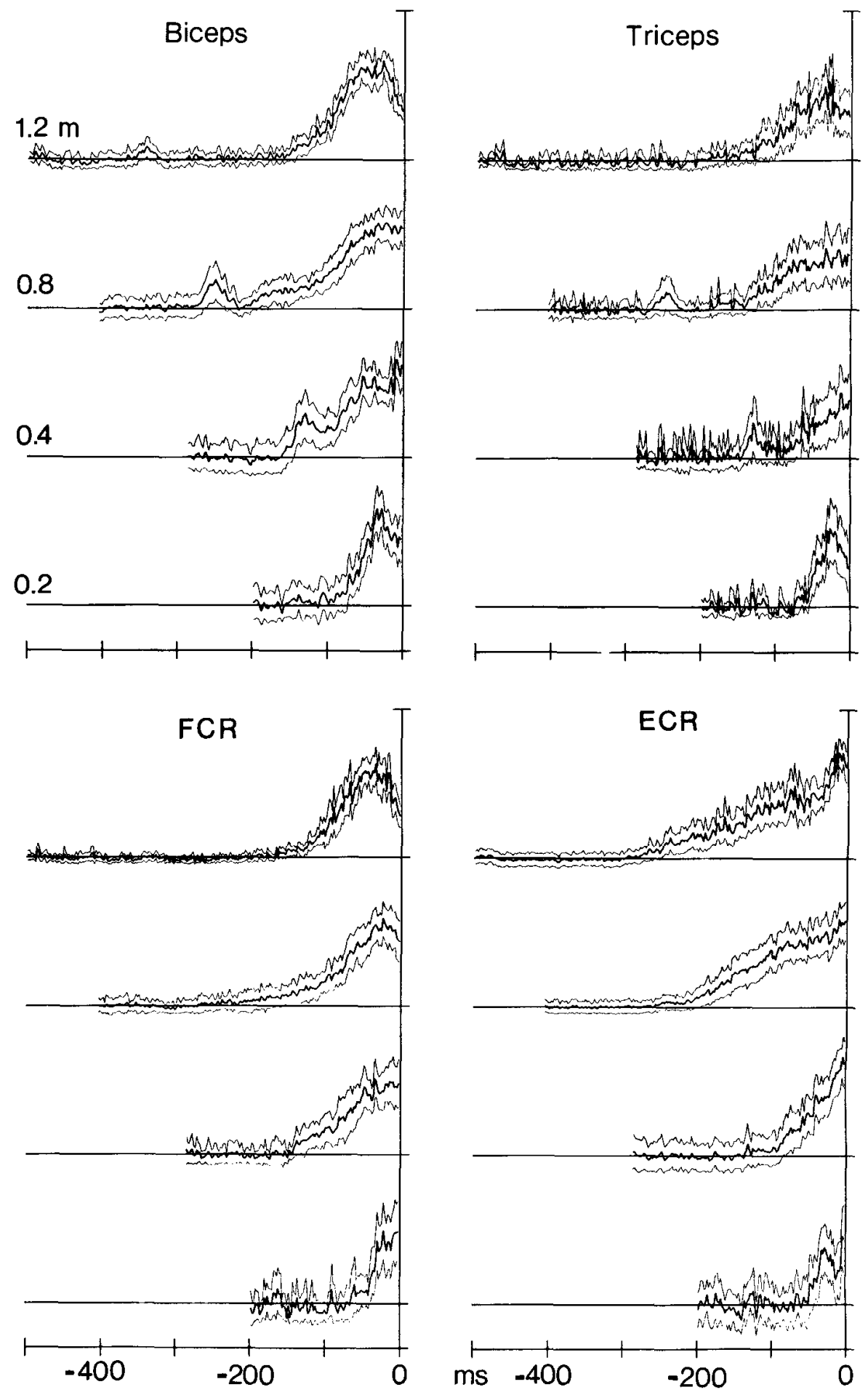

of the anticipatory activity increases with height to a larger extent than in biceps, triceps, and FCR. (The increment in duration of ECR responses amounted to $33 \%$ of the increment in duration of fall from $h=0.2 \mathrm{~m}$ to $h=1.2 \mathrm{~m}$, while the corresponding increment for biceps, triceps, and FCR amounted to about $17 \% .^{2}$ )

${ }^{2}$ One can compute the distance away from the hand when late responses begin From the values of onset time averaged across biceps, triceps, and FCR in Table 2 , one gets $0.08,0.20,0.32$, and $0.40 \mathrm{~m}$ for the heights of fall of $0.2,0.4,0.8$, and $1.2 \mathrm{~m}$, respectively.
Amplitude of the anticipatory responses

The mean amplitude of early EMG responses in biceps was computed over the 130-160 msec interval following ball release. In all experiments, this value decreased significantly with increasing height of fall. $[F(2,24)=4.8, p<0.05$, in a 3-way analysis of variance, 3 heights $\times 3$ ball masses $\times 7$ experiments A; $F(2,12)=11.6, p<0.005$, in 4 experiments B. The $F$ test for the effect of ball mass was not significant.]

The mean amplitude of late anticipatory responses (computed 


\begin{tabular}{lllcccc}
\hline Table & 2. & Time of onset of late anticipatory responses \\
\cline { 4 - 7 }$h$ & & $d$ & \multicolumn{5}{c}{ EMG duration (msec) } \\
\cline { 4 - 7 }$(\mathrm{m})$ & $n$ & $(\mathrm{msec})$ & Biceps & Triceps & FCR & ECR \\
\hline 0.2 & 21 & 198 & 53 & 44 & 34 & 39 \\
& & $(12)$ & $(17)$ & $(12)$ & $(20)$ & $(29)$ \\
0.4 & 33 & 284 & 80 & 104 & 64 & 50 \\
& & $(13)$ & $(30)$ & $(55)$ & $(30)$ & $(22)$ \\
0.8 & 33 & 402 & 96 & 96 & 76 & 128 \\
& & $(7)$ & $(27)$ & $(43)$ & $(37)$ & $(52)$ \\
1.2 & 12 & 497 & 106 & 94 & 82 & 139 \\
& & $(19)$ & $(17)$ & $(38)$ & $(27)$ & $(66)$
\end{tabular}

The time of onset was computed relative to the time of impact. Values are means $( \pm 1$ SD) over all experiments. The results of drops from $0.2 \mathrm{~m}$ have also been included since early and late components coincide in such cases.

over the $50 \mathrm{msec}$ interval preceding ball impact) also varied in an orderly manner with experimental condition in every muscle. Specifically, it increased significantly $(p<0.05)$ with increasing height of fall or mass of the ball (see Fig. 3). The analysis of variance also revealed significant first-order interactions between these 2 factors. However, a more parsimonious model can also account for the scaling in amplitude of late responses. Figure 5 demonstrates the existence of a strong linear relation between the mean amplitude of late responses in biceps and the theoretical momentum of the ball at impact time. Data points were obtained by averaging the results (after normalization) of all 4 experiments performed according to protocol B of Table 1. Similar relations also apply to the other muscles investigated. On average, a simple linear regression of mean EMG amplitudes on momentum explains $71 \pm 19 \%$ of the variance of the former in individual experiments ( 11 experiments and 4 muscles, $n=$ 44). This proportion of explained variance was not significantly increased by including either height (or fall duration) or ball mass along with momentum in a multiple linear-regression analysis.

A further test of the adequacy of a simple model linear in momentum to predict changes in the amplitude of late EMG responses is provided by the experimental protocol A of Table 1. According to such a protocol, similar values of momentum at impact result from different combinations of height of fall and ball mass (reported in the diagonals of the matrix in Table 1). For instance, a $0.8 \mathrm{~kg}$ ball falling from $0.2 \mathrm{~m}$ has a momentum at impact close to that of a $0.6 \mathrm{~kg}$ ball falling from $0.4 \mathrm{~m}$, and is identical to that of a $0.4 \mathrm{~kg}$ ball from $0.8 \mathrm{~m}$. If momentum is the determinant of response amplitude, one should expect similar values of response in all such cases, irrespective of the specific combinations of height and mass. This prediction was indeed fulfilled, as indicated by the data of Table 3, which reports the average values $( \pm 1 \mathrm{SD})$ of late responses obtained by pooling the normalized results for biceps, triceps, FCR, and ECR in all experiments. Clearly, the average values do not differ substantially along the diagonals of the matrix.

\section{Time course of the reflex responses}

The temporal relationship between the changes in kinematic and dynamic variables following the impact and the corresponding changes in the EMG activities is illustrated in Figure 6 (representative ensemble averages from 2 subjects). At impact, the impulse of force on the hand, corresponding to the change in ball momentum, resulted in wrist and elbow torques in the

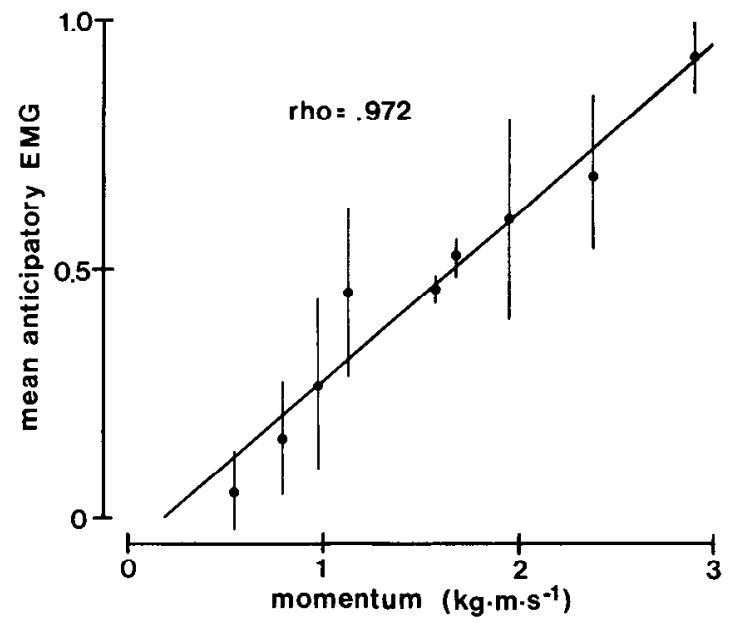

Figure 5. Linear relation between the mean amplitude of late anticipatory responses (computed over the $50 \mathrm{msec}$ interval preceding ball impact) in biceps and the theoretical momentum of the ball at impact time. Data points correspond to the mean values $( \pm 1 \mathrm{SD}$ ) of the (normalized) results of all 4 experiments performed according to protocol B of Table 1.

extensor direction. The plotted net torques ( $T_{e}$ and $T_{w}$ of equations 1 and 2; see Materials and Methods) include the contributions of both this external force and the restoring forces of muscles and connective tissue. Net torques peaked in extension at 6-8 msec after impact, and subsequently overshot their baseline towards flexion (due to the restoring forces) at 10-20 msec. These torques resulted in changes in wrist angular velocity that wcre initially towards extension (with a minimum around 10 msec) and subsequently towards flexion (with a maximum around $50 \mathrm{msec}$ ). The changes in elbow angular velocity were in the direction roughly opposite that of wrist velocity. A small initial flexion, due to the dynamic coupling with wrist angular motion, was followed by a much more pronounced extension. Mechanical oscillations at the elbow and wrist joints were damped out within about 300 msec after impact.

According to the classical notions about stretch reflexes, one would expect that the perturbation-evoked EMG responses organized reciprocally over flexor and extensor muscles, the muscles that are stretched by the perturbation being reflexly activated and the shortening muscles being relaxed (see Matthews, 1972). Instead, in agreement with our preliminary findings (Lacquaniti and Maioli, 1987), both flexor and extensor muscles at the elbow and wrist joints were consistently activated at a short latency following impact. Thus, in Figures 3 and 6 , it can be

Table 3. Amplitude of late anticipatory responses in experiment $A$

\begin{tabular}{lccc}
\multicolumn{5}{c}{ EMG amplitude at indicated $h(\mathrm{~m})$} \\
\cline { 2 - 4 } $\mathrm{m}(\mathrm{kg})$ & 0.2 & 0.4 & 0.8 \\
\hline 0.8 & 0.284 & 0.573 & 0.925 \\
& $(0.257)$ & $(0.317)$ & $(0.159)$ \\
0.6 & 0.129 & 0.338 & 0.599 \\
& $(0.226)$ & $(0.272)$ & $(0.253)$ \\
0.4 & 0.104 & 0.159 & 0.255 \\
& $(0.190)$ & $(0.164)$ & $(0.168)$ \\
\hline
\end{tabular}

Normalized values for biceps, triceps, FCR, and ECR have been averaged over all experiments $(n=7)$. Thus, each cell has mean values $( \pm 1$ SD) over 28 replicates. 

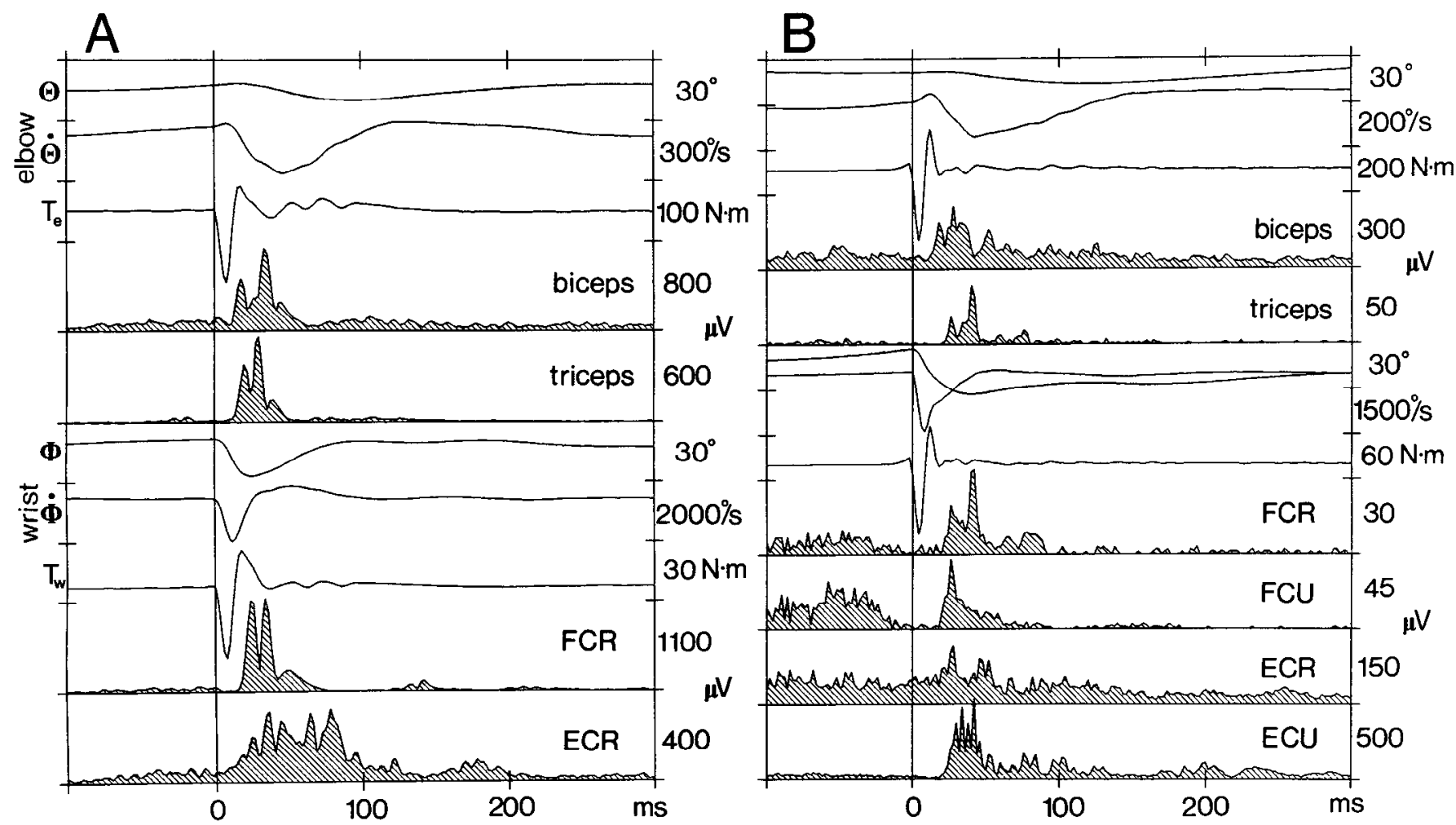

Figure 6. Time course of the reflex responses. Ensemble averages from 2 experiments ( 2 subjects): a $0.4 \mathrm{~kg}$ ball was dropped from $0.8 \mathrm{~m}$ in $A$ and from $1.2 \mathrm{~m}$ in $B$. Time of impact is indicated by the vertical line $(t=0)$. Traces from top to bottom correspond to angular position $(\Theta)$, velocity $(\dot{\Theta})$, and torque $\left(T_{c}\right)$ at the elbow, rectified EMG activity of biceps and triceps, angular position $(\Phi)$, velocity $(\dot{\Phi})$, and torque $\left(T_{w}\right)$ at the wrist, EMG activity of FCR, ECR $(A)$, FCU, ECR, and ECU $(B)$. Scales are indicated separately for each panel.

seen that the amplitude of the EMG activity of all indicated muscles increased briskly starting from about $15-20 \mathrm{msec}$. The increment of activity above the baseline was generally quite large (e.g., in all muscles in Fig. 6); in a few cases, it was significant but of small amplitude (triceps in Fig. 3, D, E, and ECR in Fig. $3 D)$.

The mean latencies of the reflex responses over all experiments $(n=99)$ were: $18 \pm 5 \mathrm{msec}$ for biceps, $23 \pm 8 \mathrm{msec}$ for triceps, $17 \pm 4 \mathrm{msec}$ for FCR, $18 \pm 2 \mathrm{msec}$ for FCU, $22 \pm 8$ msec for ECR, and $26 \pm 12 \mathrm{msec}$ for ECU. These short-latency responses were generally fractionated in multiple peaks at about $30-40 \mathrm{msec}$ and returned towards baseline within the first 40 $60 \mathrm{msec}$ after impact in both flexor and extensor muscles. Subsequent distinct increments of activity were only inconsistently observed in these experiments (performed in the presence of vision). For instance, a medium-latency activation developing over the $40-80 \mathrm{msec}$ interval can be noted in the ECR in Figure $6, A, B$, and in the FCR and biceps in Figure $6 B$. In contrast, medium-latency responses were most prominent in the experiments performed in the absence of vision (Lacquaniti and Maioli, 1989).

In order to extract the statistically significant features of the waveform of the EMG responses over the entire population of data, the following analysis was performed. The time bins over which the EMG activity remained outside the $95 \%$ confidence limits of the baseline were computed for each ensemble average. The histograms of Figure 7 were then constructed using the results from all experimental series $(n=99)$ since the specific value of the height of fall or ball mass affected only the amplitude, not the waveform, of the responses. The envelope of these histograms is very similar in all muscles investigated (including FCU and ECU, which are not shown). In fact, the EMG activity of all muscles remained significantly above baseline from about 20 to $40 \mathrm{msec}$ after impact in more than $50 \%$ of the cases. Note also the small percentage of cases $(<10 \%)$ that showed a statistically significant depression in activity within the first $40 \mathrm{msec}$.

To estimate the overall direction and amplitude of the shortlatency reflex responses, we computed their mean amplitude over the 15-40 msec interval and subtracted the baseline (see Materials and Methods). In $94 \%$ of the cases, this value was positive; on average it amounted to 13 times the baseline amplitude. In the remaining $6 \%$, it was slightly negative $(-0.19)$.

On average, the ratio between the mean values of amplitude in pairs of antagonist muscles was $82 \pm 67 \%$. Thus, it is evident that the described EMG responses cannot be accounted for by cross-talk, such as that due to volume conduction of electrical activity originating in the stretched muscles and propagating to the antagonist muscles. In fact, at the end of the experimental sessions, we also tested tendon taps on biceps and FCR with the limb in the same position as during the other trials; these stimuli elicited EMG responses in the extensor muscles whose amplitude was less than $10 \%$ of that recorded in the tapped muscle. (Similar values of cross-talk have been reported between tibialis anterior and soleus; see Gottlieb et al., 1982.)

\section{Amplitude scaling of the reflex responses}

The mean amplitude of the short-latency responses evoked by the impact generally increased with increasing intensities of the perturbation. For instance, in Figure 3, $A-C$, the amplitudes of FCR responses were 93,126 , and $143 \mu \mathrm{V}$, those of ECR re- 


\begin{tabular}{lllll}
\hline \multicolumn{5}{l}{ Table 4. Correlation between reflex amplitude and peak torque } \\
Experiment & Biceps & Triceps & FCR & ECR \\
\hline 1 & $0.906^{a}$ & $0.814^{a}$ & $0.952^{a}$ & $0.835^{a}$ \\
2 & 0.382 & 0.358 & 0.568 & 0.198 \\
3 & $0.743^{a}$ & 0.539 & $0.847^{a}$ & 0.417 \\
4 & 0.508 & $0.901^{a}$ & $0.595^{a}$ & -0.154 \\
5 & $0.689^{a}$ & $0.700^{a}$ & 0.078 & 0.217 \\
6 & 0.532 & 0.537 & $0.666^{a}$ & $0.820^{a}$ \\
7 & $0.728^{a}$ & $0.964^{a}$ & 0.468 & -0.474 \\
8 & $0.707^{a}$ & -0.373 & $0.607^{a}$ & $0.680^{a}$ \\
9 & 0.563 & 0.042 & 0.567 & 0.423 \\
10 & $0.995^{a}$ & $0.964^{a}$ & 0.432 & $0.843^{a}$ \\
11 & $0.945^{a}$ & $0.997^{a}$ & $0.998^{a}$ & $0.824^{a}$ \\
Mean & $0.781^{a}$ & $0.777^{a}$ & $0.737^{a}$ & $0.487^{a}$
\end{tabular}

The correlation coefficients $(r)$ are reported for all experiments. The mean correlation coefficients have been computed using their $Z$-transformed values: $Z=\tanh ^{-1} r$ (Kendall and Stuart, 1969).

${ }^{a}$ Statistically different from $0(p<0.05)$. [Note that the variance for the mean correlation coefficients is 11 (number of experiments) times smaller than the variance for individual experiments.]

sponses were 11,13 , and $55 \mu \mathrm{V}$, and the corresponding values of ball momentum were $1.58,2.24$, and $3.17 \mathrm{~kg} \mathrm{~m} / \mathrm{sec}$, respectively.

These results might have been expected for the responses of muscles that are stretched by the perturbation, but not necessarily for those of shortening muscles. (Had stretch reflexes been operative, the amplitude of the response in shortening muscles should presumably have decreased with increasing perturbations.) Figure 8 demonstrates the positive linear correlations obtained over the 9 ensemble averages of one experiment. The mean amplitudes of the short-latency EMG responses have been plotted as a function of the peak extensor torques at the elbow and wrist joints. (Such peak torques are strongly correlated with the value of ball momentum at impact.) Note that the reflex responses scale over the entire range of variation of the peak torqucs.

Table 4 reports the list of the correlation coefficients $(r) \mathrm{ob}$ tained in all experiments. Despite the variability of the results, the overall trend was in the direction described above. In fact, in $93 \%$ of the cases $r$ was positive (although it was not statistically different from 0 in about half of them). Furthermore, the mean values of the correlation coefficients computed over all experiments were significantly positive for all muscles.

\section{Mechanical behavior of the arm}

Ball impact results in mechanical oscillations at the elbow and wrist joints whose amplitude and time course vary as a function of the applied perturbation (see Figs. 3 and 6). One question raised by the reported observations concerns the modality and extent to which neural and mechanical factors contribute to damp such oscillations.

First, the perturbation is resisted by restoring forces that result from the intrinsic viscoelastic properties of muscles. On this subject, it is known that the static and dynamic components of muscle stiffness increase with the mean muscular tension (see Houk and Rymer, 1981). One might therefore suppose that anticipatory activation presets muscle viscoelastic parameters to a value that is an increasing function of the momentum at impact (see Fig. 5).

On the other hand, the reflex responses evoked by the per-
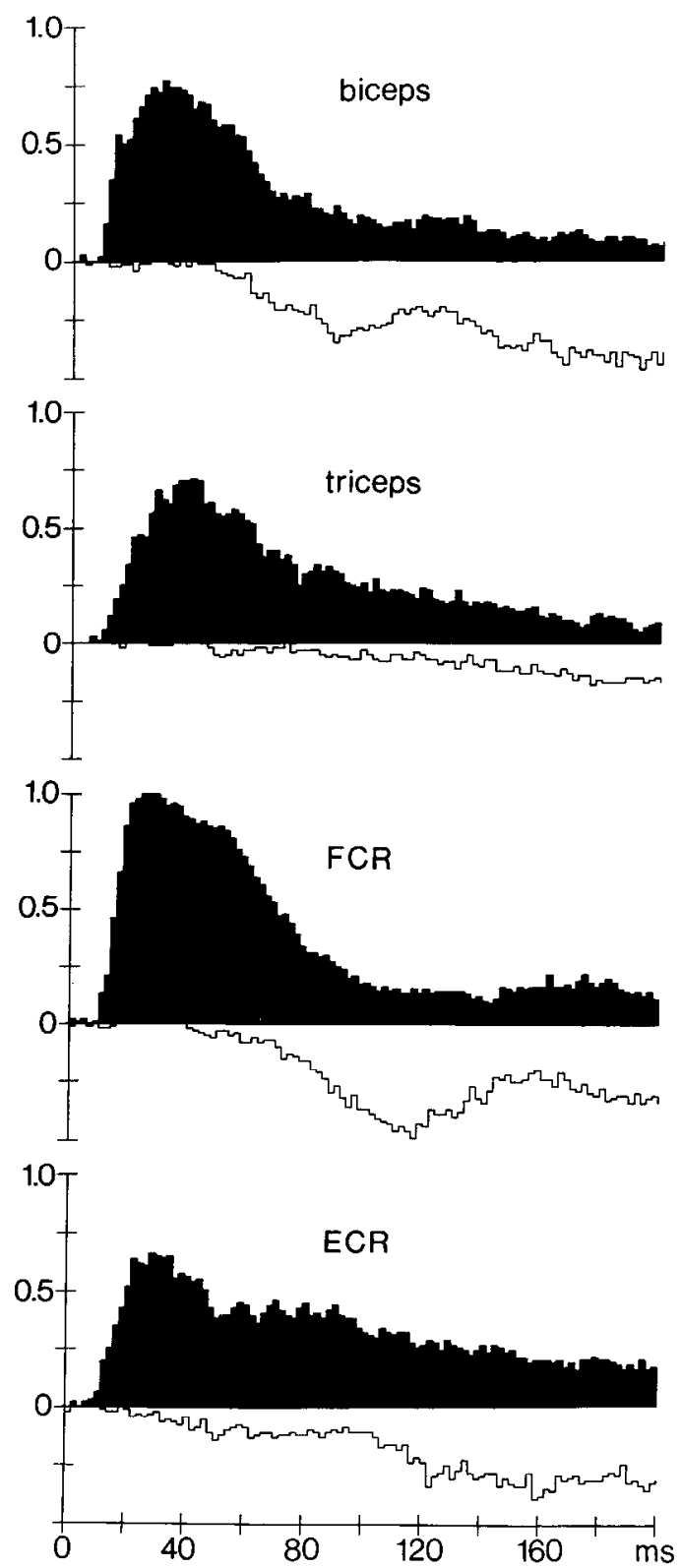

Figure 7. Time histograms of the EMG responses evoked by the impact which were significantly different from the baseline. The time bins ( $2 \mathrm{msec}$ ) in which the EMG activity of the indicatcd muscles was significantly $(p<0.05$ ) above (black) or below (white) the prereflex baseline are plotted for all ensemble averages $(n=99) . t=0$ corresponds to the time of impact. Note that the envelope of these histograms is very similar in all muscles.

turbation might also contribute to the overall limb stiffness and viscosity. However, while feedback contributions due to stretch reflexes organized according to the classical principle of reciprocal innervation have been extensivcly studied (see Agarwal and Gottlieb, 1984), the potential contributions from the reflex coactivation observed in the present experiments must be investigated.

The exact assessment of the neural and mechanical contributions to limb stiffness would require direct measurement of the changes in length and tension of individual muscles, along with their patterns of neural activation. Clearly, these measurements were not feasible in the present experiments. Never- 
Figure 8. Amplitude scaling of the reflex responses. Linear correlations between the mean amplitudes of the shortlatency EMG responses (over the 1540 msec interval after impact) and the peak extensor torques at the elbow and wrist joints. Data points correspond to the results obtained in the 9 ensemble averages of one experiment ( filled circles for biceps and FCR, open circles for triceps and ECR).
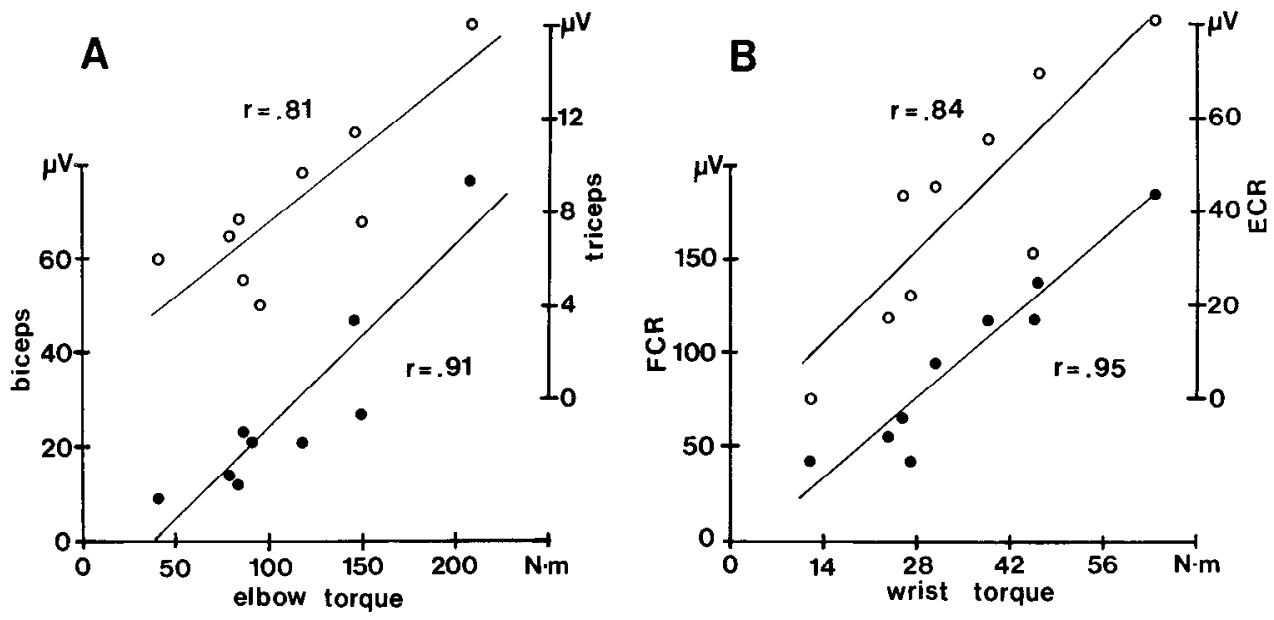

theless, a rough estimate of the contributions due to the observed patterns of muscle activation can be obtained by means of simulation studies based on some simplifying assumptions. The first assumption is that the extensor muscles of the elbow and wrist joints are coactivated with the corresponding flexor muscles so as to produce equal and opposite joint torques. If so, the antagonist muscles would cooperate to augment joint angular stiffness and viscosity. The time course of such changes in stiffness and viscosity is dictated by the filtering properties of muscles and can be estimated by convolving the changes in EMG activity with the known muscle twitch profile. Scaling factors for the changes in stiffness and viscosity are found by fitting simulated kinematics to the experimental data (see Appendix 1 for details).

Representative results of this analysis are shown in Figure 9. The EMG traces assigned to elbow and wrist "equivalent" muscles are those experimentally recorded in biceps and FCR, respectively. (Although this choice is arbitrary, recall that the envelope of the EMG responses did not differ markedly among the muscles investigated.) Stiffness (and viscosity) at the elbow and wrist change with the illustrated time course. Note that the model predicts a significant modulation of joint stiffness and viscosity and, in particular, a large increment due to reflex coactivation. Thus, the peak values of joint stiffnesses were reached about $80-100$ msec after impact. On average, such peak values

Table 5. Joint stiffness and viscosity coefficients

\begin{tabular}{cccll} 
Experiment & $\begin{array}{l}K_{\theta \theta} \\
(\mathrm{N} \mathrm{m} / \mathrm{rad})\end{array}$ & $\begin{array}{l}K_{\theta *} \\
(\mathrm{~N} \mathrm{~m} / \mathrm{rad})\end{array}$ & $\begin{array}{l}K_{\text {s }} \\
(\mathrm{N} \mathrm{m} / \mathrm{rad})\end{array}$ & $\begin{array}{l}C \\
(\mathrm{sec})\end{array}$ \\
\hline 1 & 39.6 & 9.7 & 33.3 & 0.028 \\
2 & 30.1 & 8.1 & 40.3 & 0.044 \\
3 & 83.3 & 31.4 & 39.4 & 0.055 \\
4 & 37.3 & 10.3 & 30.0 & 0.045 \\
5 & 49.2 & 11.5 & 63.2 & 0.016 \\
6 & 49.8 & 20.0 & 20.5 & 0.105 \\
7 & 36.3 & 19.7 & 22.0 & 0.038 \\
8 & 35.8 & 8.6 & 33.2 & 0.051 \\
9 & 87.2 & 41.9 & 61.2 & 0.050 \\
10 & 105.3 & 41.7 & 49.2 & 0.086 \\
11 & 44.7 & 20.8 & 26.4 & 0.039 \\
Mean & 54.4 & 20.3 & 38.1 & 0.051
\end{tabular}

Stiffness and viscosity coefficients at the elbow and wrist joints from all experiments involving falls of $0.4 \mathrm{~kg}$ ball from $0.8 \mathrm{~m}$. were 2.6 and 6.7 times larger than the corresponding values at impact for the elbow and wrist joints, respectively. The resulting kinematics (thick curves) is plotted superimposed on the experimental data (thin curves). Clearly, the model adequately fits the data. On the average, the mean absolute deviation from experimental angular velocity computed over $200 \mathrm{msec}$ after impact was $6 \pm 2 \%$ of the peak angular velocity over all sets of data $(n=99)$. However, the high-frequency oscillations that are present in the data arc not reproduccd by the modcl. Such oscillations might, in fact, be extraneous to true limb kinematics, being related instead to the mechanical resonance of the goniometer. However, the possibility that they result from patterns of muscle activities other than simple coactivation cannot be discounted.

As mentioned above, the fitting procedure yields estimates of the peak values of stiffness and viscosity coefficients (reached 80-100 msec after impact). The values obtained in all experiments involving $0.8 \mathrm{~m}$ drops of a $0.4 \mathrm{~kg}$ ball are listed in Table 5 . Table 6 shows the average values $( \pm 1 \mathrm{SD})$ of the stiffness coefficients $K_{\text {क्ष }}$ computed over all experiments A. Despite the sample variability, such values exhibit the same trend as previously found for the amplitudes of anticipatory and reflex EMG responses: The coefficients $K_{\Phi \Phi}$ increase linearly with the momentum of the ball at impact $(\rho=0.93)$. Similar trends exist for the other stiffness coefficients $\left(K_{\theta \theta}\right.$ and $\left.K_{\theta \Phi}\right)$.

\section{Hand stiffness}

Stabilization of elbow and wrist angular positions during catching is but one of the factors influencing stable prehension. In fact, the dynamic interaction of the limb with the ball is affected by the resistance opposed by the hand to a linear displacement

Table 6. Stiffness coefficients $K_{\phi \phi}$ in experiment $A$

\begin{tabular}{lccc}
\multirow{2}{*}{$(\mathrm{kg})$} & \multicolumn{4}{l}{$K_{\Phi \Phi}(\mathrm{N} \mathrm{m} / \mathrm{rad})$ at indicated $h(\mathrm{~m})$} \\
\cline { 2 - 4 } 0.8 & 0.2 & 0.4 & 0.8 \\
\hline \multirow{2}{*}{0.6} & 46.7 & 54.5 & 61.9 \\
& $(27.5)$ & $(33.6)$ & $(36.2)$ \\
0.4 & 27.8 & 40.2 & 50.0 \\
& $(13.2)$ & $(10.1)$ & $(39.4)$ \\
& 25.9 & 39.4 & 39.5 \\
& $(21.5)$ & $(15.7)$ & $(14.0)$
\end{tabular}

Mean values $( \pm 1 \mathrm{SD})$ computed over all experiments $\mathrm{A}(n=7)$. 

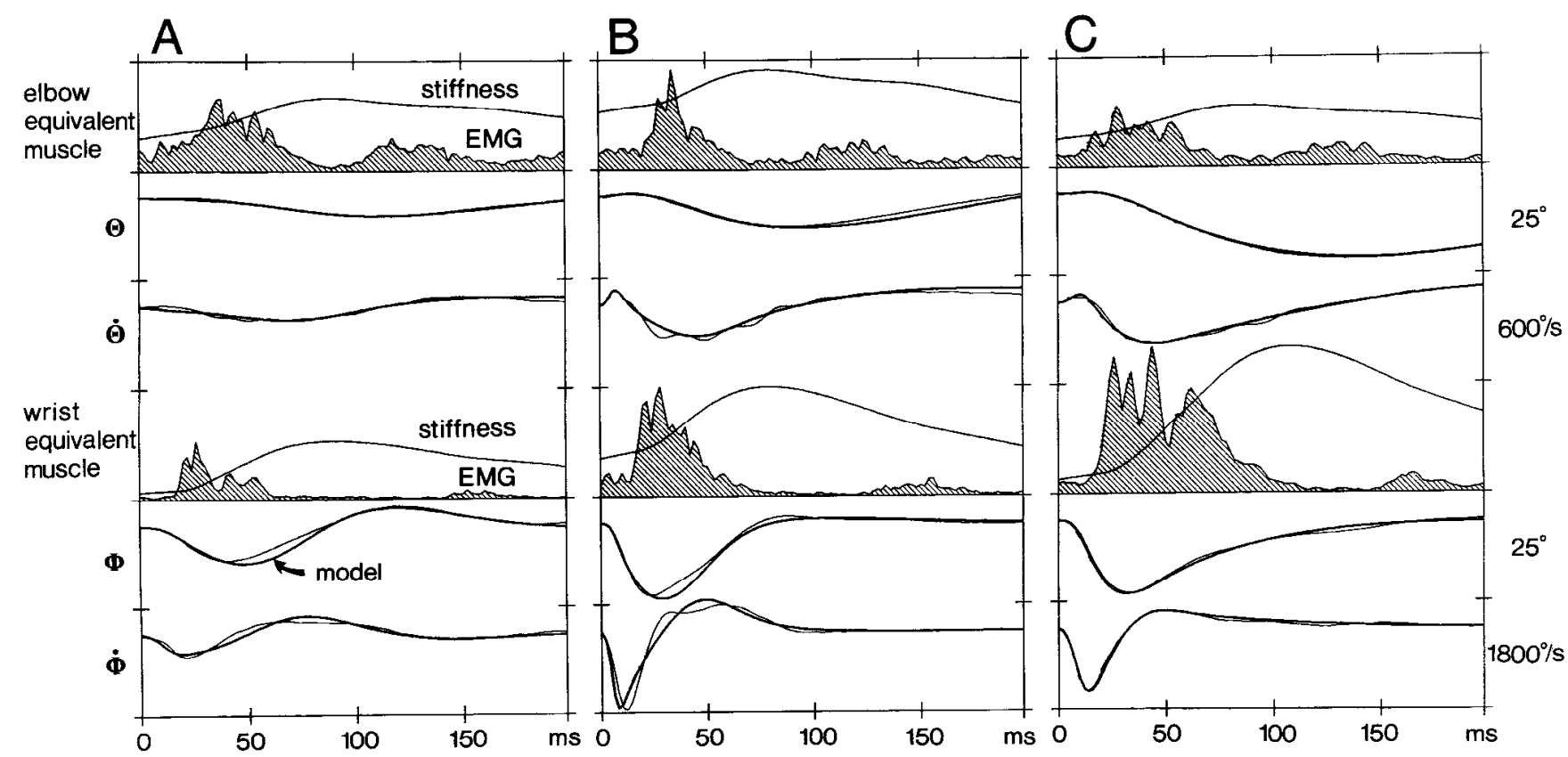

Figure 9. Simulations of the mechanical behavior of the limb. The results obtained in 2 experiments ( $A$ and $B$, and $C$, respectively) were modeled using a system with linear, time-varying viscoelastic coefficients. A $0.2 \mathrm{~kg}$ ball was dropped from $0.4 \mathrm{~m}$ in $A$, a $0.4 \mathrm{~kg}$ ball from $1.2 \mathrm{ml}$ in $B$, and a $0.8 \mathrm{~kg}$ ball from $0.8 \mathrm{~m}$ in $C$. The EMG traces assigned to elbow and wrist "equivalent" muscles are those experimentally recorded in biceps and FCR, respectively. Stiffness (and viscosity) at the elbow and wrist change with the illustrated time course. Note that the model predicts a significant modulation of joint stiffness and viscosity, and in particular a large increment due to reflex coactivation. The resulting kinematics (thick curves) is plotted superimposed on the experimental data (thin curves).

along the direction of fall. We must then address the question of whethcr and how hand stiffness changes during catching.

We have seen that anticipatory and reflex muscle coactivations result in a time-varying modulation of the angular stiffness and viscosity at the elbow and wrist joints that can account for the observed kinematics following impact. The value of hand stiffness, however, depends not only on the joint angular stiffnesses but also on the geometrical configuration that the limb takes in space at any instant (Hogan, 1985; Mussa-Ivaldi ct al., 1985). Since limb movement occurs both before and after impact (see Fig. 3), changes in hand stiffness need not parallel a priori the changes in joint stiffnesses. Thus, we computed the (time-varying) matrix of hand stiffness coefficients starting from the joint stiffness matrix previously found and the measured limb trajectory (see Appendix 2). The resistance opposed by the hand to a virtual vertical displacement corresponds then to the components $R_{x y}, R_{y y}$ of such hand stiffness matrix (equation 14).

The stick diagrams of Figure $10 \mathrm{~A}$ depict the limb trajectory over the $200 \mathrm{msec}$ interval centered on impact time in a representative case. For clarity, these diagrams have been offset along the oblique axis proportionally to elapsed time. Limb end point corresponds to the third metacarpophalangeal joint, where impact occurs. The arrows departing from this point denote the vectors $\left[R_{x y} R_{y y}\right]{ }^{3}$ It can be seen that the hand is raised towards the incoming ball as a result of elbow and wrist flexion occurring during the last $100 \mathrm{msec}$ before impact. After impact, the hand is rapidly deflected downwards.

Throughout the duration of limb movement, hand stiffness undergoes substantial changes in magnitude, as indicated by the variation in the length of the arrows (the modulus of the stiffness

\footnotetext{
${ }^{3}$ These vectors can be interpreted as the force $F$ at the end point for a unit
} virtual displacement along the vertical $(\delta x=0, \delta y=1$ in equation 6$)$. vector, $\left.\left[R_{x y}^{2}+R_{y y}^{2}\right]^{1 / 2}\right)$. Also their inclination over the horizontal (the argument of the stiffness vector, $\tan ^{-1}\left[R_{y y} / R_{x y}\right]$ ) changes somewhat. In particular, both the modulus and the argument increase throughout the anticipatory movement.

The changes in magnitude can be best appreciated in Figure $10 \mathrm{~B}$. In each set of data, the modulus of the stiffness vector was computed over the $600 \mathrm{msec}$ interval centered on impact. Grand averages $( \pm 1 \mathrm{SD})$ were then constructed using the results of all cxpcriments $(n=99)$ scaled to their maximum. The time course of the average changes in hand stiffness is multiphasic. Stiffness rises sharply above baseline about $50 \mathrm{msec}$ before impact and reaches a first maximum at impact. A second, broader peak at $130 \mathrm{msec}$ after impact is separated from the first by a trough at 20 msec.

We may now seek to dissect the contribution due to the changes in neural activity from that due to the changes in limb geometry related to the movement. To this end, we calculate the theoretical time course of the changes in hand stiffness which would be observed in case of a constant level of muscle activity. The continuous curve in Figure $10 \mathrm{C}$ corresponds to this hand stiffness due solely to geometric factors. It has been calculated assuming that muscle activity is kept at the baseline level throughout. (However, a similar time course is obtained by choosing other values of constant muscle activity.) The dashed curve has been obtained by subtracting the former curve from the net hand stiffness of Figure $10 \mathrm{~B}$. Therefore, it corresponds to an estimate of the additional contribution to net hand stiffness due to time-varying modulation of muscle activity. Remarkably, both "geometrical" and "muscle" stiffness curves exhibit a peak at the time of impact, indicating a synergism between anticipatory movement of the limb and muscle stiffening. The deflection of the hand produccd by the impact results in a substantial drop of "geometrical" stiffness that recovers only slowly. 

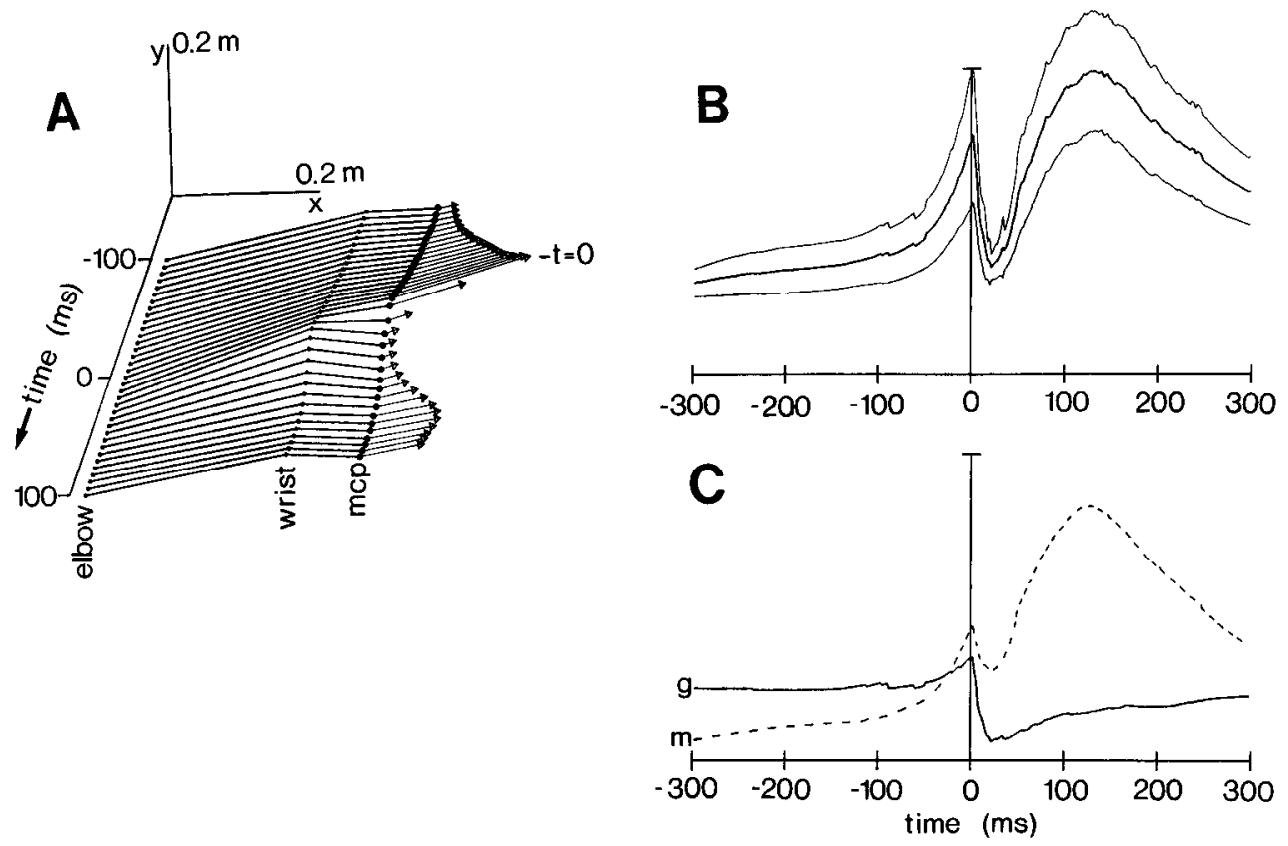

Figure 10. Time course of hand stiffness. A, Stick diagrams plotting the limb trajectory over the $200 \mathrm{msec}$ interval centered on impact time in a representative ensemble average. These diagrams have been offset along the oblique axis proportionally to elapsed time (time resolution is 6 msec). Limb end point corresponds to the third metacarpophalangeal joint, where impact occurs. The arrows departing from this point denote the vectors corresponding to the resistance opposed by the hand to a virtual vertical displacement (equation 14). The components $R_{x y}$ of such vectors are plotted along the $x$-axis, while the components $R_{y y}$ are plotted along the $y$-axis. $B$, The overall changes in magnitude of the vectors. In each set, the modulus of the stiffness vector $\left.\left(\left[R^{2}{ }_{x y}+R^{2}\right]_{y y}\right]^{\prime / 2}\right)$ was computed over the $600 \mathrm{msec}$ interval centered on impact. Grand averages $( \pm 1 \mathrm{SD})$ were then constructed using the results of all experiments $(n=99)$ scaled to their maximum. $C$, The continuous curve corresponds to the hand stiffness due solely to geometric factors. It has been calculated assuming that muscle activity is kept at the baseline level throughout. The dashed curve has been obtained by subtracting the former curve from the net hand stiffness of $B$ and therefore corresponds to an estimate of the additional contribution to net hand stiffness due to time-varying modulation of muscle activity.

On the contrary, the drop in "muscle" stiffness recovers much more rapidly (due to reflex coactivation) and the subsequent large increment offsets the decrement in "geometrical" stiffiness completely.

\section{Discussion}

We set out to address the following questions. (1) Which parameters of ball and limb motion are the muscle responses related to? (2) What roles do sensory information and central set play in tuning the responses? (3) What is the functional significance of the responses in stabilizing limb posture during catching? We shall take up these issues for both the anticipatory and reflex responses.

\section{Anticipatory responses}

Early and late components could be identified in the EMG anticipatory responses. Early responses were produced at a roughly constant latency (about $130 \mathrm{msec}$ ) from the release of the ball, corresponding to a visual reaction time for a highly compatible stimulus-response relation (Georgopoulos et al., 1981; Soechting and Lacquaniti, 1983). Their amplitude was inversely proportional to the height of fall. Thus, they presumably represent an alertness reaction that already incorporates an estimate of the duration of fall $(d)$ : The shorter the time available for the preparation to the impact, the larger the size of the population of $\alpha$-motoneurons recruited within the reaction time. Since this recruitment lasts only $30-50 \mathrm{msec}$, it bears no direct motor consequences on catching except for the smallest drops. It might, instead, be considered germane to covert cognitive processes related to motor preparation.

Late EMG responses comprised the major build-up of activity prior to impact. Their onset and duration with respect to the time of impact varied little from case to case (except for ECR responses, whose duration increased appreciably with the size of the drop). This fact and the occurrence of the peak of activity just prior to impact indicate that this motor output is precisely timed on $d$. The latter parameter might be estimated a priori (using solely information about the height of fall), and the time course of the responses might be entirely preset. If so, one would expect to find similar responses when the time of ball release is signaled to the subject but visual feedback of the motion is prevented (see McKinley and Smith, 1983). Data presented in the companion paper (Lacquaniti and Maioli, 1989) contradict this expectation by demonstrating that the very existence of anticipatory responses is contingent upon the presence of vision. From this we conclude that the time course of the late responses is probably controlled on line based on an estimate of instantaneous time-to-contact $\left[t_{c}(t)\right.$, time remaining before impact]. Thus, the nature of the dynamic visual information that might afford the estimate of $t_{c}(t)$ must be considered.

Optical flow field (i.e., the field of the instantaneous positional velocities of the image on the retina) is the primary source of visual information from which to compute $t_{c}(t)$ (Gibson, 1966). Lee (1980) has shown that, for an object in uniform motion perpendicular to the projection plane of a stationary eye, $t_{c}(t)$ is univocally specified by the rate of dilation $[\tau(t)]$ of the retinal image. However, when the approach is uniformly accelerated, 
as in the present experiments, $\tau(t)$ overestimates $t_{c}(t)$. Furthermore, since the path of the ball is at an angle from the sightline, more than one component of the optical flow (e.g., dilation and translation) presumably contributes to specify $t_{c}(t)$.

Optical flow is thought to be computed from the short-range motionanalyzing processes, which rely on directionally sensitive motiondetecting units, such as those of area MT, and which operate on zerocrossing maps (sce Ullman, 1986). One possible way to estimate time-to-contact using information from the optical flow is by solving the equation $t_{c}(t)=d-v(t) / g$, where $v(t)$ is the instantaneous velocity of the image and $g$ is the acceleration of gravity. Although in our experiments eye movements were not monitored, other data seem to indicate that they too might contribute in collecting information about $t_{c}(t)$ (Sharp and Whiting, 1975). For instance, the summation of the efferent copy of the command encoding eye velocity and of the retinal velocity error could provide a signal proportional to the target velocity.

The other major finding concerning the late EMG responses is that their mean amplitude is linearly related to the theoretical momentum of the ball at time of impact. Differently stated, this result implies that subjects scale the amount of anticipatory muscle contraction in proportion to the impulse of force $(I=$ $\Delta m v$ ) they expect to receive. Similarly, Karst and Hasan (1987) recently argued that the time integral of the EMG activity of a muscle that brakes a fast voluntary movement is related to a power function of the peak kinetic energy of the limb.

How does a subject predict ball momentum at impact? We have discussed above the availability of velocity information through vision. As for the other term in the expression for momentum-namely, the ball mass - the following considerations are in order. First, we do not believe that the power laws that apply to psychophysical scaling of lifted weights (see Stevens, 1975) are pertinent here. Indeed, the perccptions of gravitational mass and inertial mass are inextricably confounded in lifting. By contrast, during catching it is the inertial mass of the ball that is primarily experienced at impact. Experiments conducted under microgravity indicate that mass discrimination does differ from weight discrimination, although the specific differences may depend on the experimental conditions (Ross et al., 1986). It is conceivable that in the present experiments an internal model of the ball's properties is initially used to set the amplitude of muscle anticipatory contraction, while kinesthetic and cutaneous information obtained during the act of catching is subsequently utilized to calibrate this and other motor parameters (see Lacquaniti and Maioli, 1989). One might also conceive, as an alternative to separate processes for estimating mass and velocity, a single process that scales momentum as one unitary variable and tunes motor actions accordingly.

In summary, it appears that no single sensory-perceptual process can be pinpointed as the source of information for estimating either time-to-contact or momentum. These estimates, then, might be included among the "affordances" in the ecological sense given by Gibson (1966), which are determined by the fit between the properties of the environment and the properties of the organism's action system. The numerous degrees of freedom inherent in this fit would thus make the specific solutions context-dependent.

One final point on the organization of the anticipatory responses must be made concerning the pulse-height control hypothesis (see Freund and Büdingen, 1978; Gordon and Ghez, 1987), which states that the control of either single-joint movements or isometric contractions matching targets of different amplitude is achieved simply by scaling the amplitude of an otherwise invariant EMG waveform (see, however, Brown and Cooke, 1984). The advantage of such a strategy is a reduction in the complexity of the control problem. It should be noted, however, that this hypothesis applies only loosely to the anticipatory responses observed during catching. Indeed, their amplitude scales with that of the expected perturbation, but their waveform also changes with the height of fall (particularly in wrist extensors).

\section{Reflex responses}

The EMG reflex responses evoked by ball impact consisted in a short-latency, brief coactivation of flexor and extensor muscles. Although the direction of the changes in elbow angular velocity induced by the impact was opposite that of the corresponding changes in wrist angular velocity, the pattern of the reflex responses of elbow and wrist muscles was very similar. These responses tended to be graded, their mean amplitude in both flexor and extensor muscles generally increasing with increasing intensities of impact.

This pattern contrasts sharply with that traditionally ascribed to stretch reflex responses. Indeed, when normal subjects resist perturbations applied to their limbs by a torque motor, the short-latency EMG responses evoked in antagonist muscles are reciprocally organized, the direction of the responses being uniquely related to the direction of the preceding changes in the angular velocity (Dufresne et al., 1979; Gottlieb and Agarwal, 1980).

The first question we must address is whether the described pattern of reflex coactivation represents an aberrant response solely due to the particular nature of the mechanical perturbation and devoid of functional significance, or if it is a response that is centrally preset to suit the needs of limb stabilization.

In favor of the first point of view, it could be argued that the impact of the ball on the hand produces a large elastic wave that propagates through soft tissue and bone and activates Ia afferents from both flexor and extensor muscles, thereby exciting the corresponding $\alpha$-motoneuron pools via homonymous connections. For instance, spread of vibration waves has been invoked to account for the sporadic cases of appreciable reflex coactivation of antagonist muscles following tendon taps in normal adults (Gurfinkel and Paltsev, 1973; Gottlieb et al., 1982; Burke et al., 1983).

We can rule out that this mechanism accounts for the reflex coactivation we observed during catching on the basis of the following considerations. The amplitude of the vibration wave over the dorsal side of the limb is considerably attenuated with respect to that over the volar side on which impact occurs (sce Burke et al., 1983). Thus, in contrast to what may happen during tendon taps involving quasi-isometric conditions, during catching any effect due to vibration would presumably be offset in Ia afferents from shortening muscles because of the high angular velocities reached after impact (see Fig. 6). Thus, one would expect that the $\alpha$-motoneurons to the shortening muscles would be disfacilitated due to muscle spindle unloading, and would be inhibited via the classical Ia reciprocal connections with the antagonists (see Baldissera et al., 1981). Note further that we were unable to elicit appreciable reflex coactivation of elbow and wrist muscles by means of tendon taps in our subjects.

It is also very unlikely that the responses elicited by ball impact represent the "shortening reactions" that have been described in both normal subjects and, more commonly, in spasticity and rigidity (see Andrews et al., 1972; Ebner et al., 1982). 
In fact such slow contractions develop at a long latency, asynchronously with respect to the contraction of the stretched muscle.

Rather, we believe that reflex coactivation is centrally preset during the catching task. One hypothesis is that the adequate stimulus is, in fact, muscle stretch, as for stretch refiex responses, but that in preparation for catching, or for other actions involving equivalent demands of limb stabilization, the reflex responses can be preset by central mechanisms gating a pattern of coexcitation of $\alpha$-motoneurons of antagonist muscles in lieu of the more usual reciprocal inhibition, for instance, by opening spinal pathways that are normally closed. This hypothesis would imply that the adequate stimulus can excite fast-conducting muscle (Ia) or tendon (Ib) afferents which have oligosynaptic excitatory connections with both the $\alpha$-motoneurons of the stretched muscles and those of the antagonist shortening muscles. In this context, it should be recalled that, although reciprocal inhibition is firmly established as the primary pattern of spinal interneuronal connection between the $\alpha$-motoneuron pools of antagonist muscles, stimulation of both $\mathrm{Ia}$ and $\mathrm{Ib}$ afferents from limb muscles in cat can also evoke widespread coexcitation of antagonist $\alpha$-motoneurons (Eccles et al., 1957; Willis et al., 1966; Watt et al., 1976; Jankowska et al., 1981). This coexcitation of antagonists is oligosynaptically mediated by lamina V-VI interneurons that are shared by both Ia and Ib afferents from several muscles acting on different limb joints (Jankowska et al., 1981). Since it is known that the excitability of these interneurons, similarly to that of Ia inhibitory interneurons, can be extensively modulated by descending tracts, as well as by joint and cutaneous afferents, one might surmise that switching from the spinal pathways of reciprocal inhibition to those of coexcitation can, indeed, occur. Note that other instances of gating of spinal reflexes are well known, particularly those involving Ia (Schomburg and Behrends, 1978) or cutaneous (Grillner, 1981) inputs during locomotion.

Although group I afferents are most likely involved in the short-latency responses we described, contributions by group II afferents (from skin, joints, and muscles) cannot be ruled out. In particular, in man, low-threshold skin afferents have conduction velocities that are only about $15 \%$ slower than those of group I afferents (Burke et al., 1983) and can significantly affect the excitability of $\alpha$-motoneurons during the slow rise time of the composite EPSPs due to group I volleys (Delwaide et al., 1981).

\section{Stabilization of limb posture}

We next address the functional significance of the described muscle responses vis-à-vis the problem of stabilizing the limb during catching. It could be argued that the mechanical behavior of the arm is dominated by its resting properties. Specifically, the restoring torques after impact could result mostly from the intrinsic viscoelastic properties of the muscles at the basal level of activity, while the changes in such activity due to anticipatory and reflex responses would be largely noninfluential. For instance, it has often been suggested that the load-compensating capabilities of short-latency stretch reflexes are rather poor because of the low loop gain compatible with stability in the presence of the neural feedback delays. The problem, then, is whether reflex coactivation can do any better.

To address this issue, we have modeled the behavior of the arm as a system with linear, time-varying viscoelastic coefficients (Lacquaniti and Soechting, 1986). The time course of the changes in angular stiffness and viscosity coefficients was constrained to follow a filtered version of the changes in EMG activity experimentally measured. Admittedly, this model neglects aspects of the system, such as muscle and neural nonlinearities, that might be relevant. Nevertheless, it does reproduce the main features of experimentally observed limb kinematics (Fig. 9). Furthermore, the estimated peak values of elbow angular stiffness and viscosity are in good agreement with those reported in studies in which subjects resist load perturbations (Zahalak and Heyman, 1979; Lacquaniti et al., 1982; MussaIvaldi et al., 1985). (As for the wrist joint, the only available values that we are aware of pertain to relaxed conditions, and these are much lower than the present ones (Lakie et al., 1984).] Thus, our tentative conclusion is that the observed changes in EMG activity preceding and following the impact may indeed contribute significantly to modulate limb compliance throughout the act of catching. If so, the import of the modulation of joint compliances in the context of the dynamic interaction between the hand and the falling ball should be evaluated. This interaction is dictated by the instantaneous value of the stiffness (and viscosity) at the hand, and therefore depends not only on the corresponding values of the joint angular stiffnesses but also on the geometrical configuration of the limb in space (Hogan, 1985).

The analysis of this problem yielded 2 relevant observations. First, anticipatory movement and anticipatory muscle stiffening are synergistic in building up resistance of the hand to the ball's impact. In fact, they contribute significantly to increasing the magnitude of the vector corresponding to the resistance opposed by the hand to a vertical displacement and to orienting the direction of this vector closer to that of the impact. The second point is that the time course of the contribution to the net hand stiffness due to the reflex responses is appropriate to counteract the decrement due to the sudden extension of the limb after the impact.

In conclusion, we have demonstrated that the task of catching involves complex preparatory changes in motor set so as to produce muscle responses that are finely tuned to the physical parameters of ball impact. These responses might be instrumental for stabilizing limb posture by taking advantage of the intrinsic load-compensating properties of active muscle, which do not incur the conduction delays of stretch reflex feedbacks.

\section{Appendix 1}

\section{Mechanical model of limb kinematics}

The model described in Lacquaniti and Soechting (1986) is here extended to include time-varying parameters.

In equations 1 and 2 of Materials and Methods the net torques acting at the elbow $\left(T_{c}\right)$ and wrist $\left(T_{w}\right)$ are the resultant of all external and internal contributions:

$$
\left[\begin{array}{l}
T_{e} \\
T_{u^{\prime}}
\end{array}\right]=\underline{\mathrm{T}}^{\prime}+\underline{\mathrm{T}}_{v}+\underline{\mathrm{T}}_{g},
$$

where $\underline{T}^{\prime}$ is the torque due to externally applied forces (ball impulsive impact), $\underline{T}_{v}$ is the viscoelastic contribution of muscles, and $\underline{T}_{g}$ is the component required to balance the gravitational terms in equations 1 and 2 (underbars denote vector quantities). $\underline{T}^{\prime}$ can be computed from impact parameters. $\underline{T}_{v}$ is estimated as

$$
\begin{aligned}
\underline{\mathrm{T}}_{v}= & -\left[\begin{array}{ll}
K_{\theta \theta}(t) & K_{\theta \Phi}(t) \\
K_{\theta \phi}(t) & K_{\Phi \Phi}(t)
\end{array}\right]\left[\begin{array}{l}
\Delta \Theta \\
\Delta \Phi
\end{array}\right] \\
& -C\left[\begin{array}{ll}
K_{\theta \theta}(t) & K_{\theta \Phi}(t) \\
K_{\theta \phi}(t) & K_{\Phi \Phi}(t)
\end{array}\right]\left[\begin{array}{c}
\dot{\Theta} \\
\dot{\Phi}
\end{array}\right]
\end{aligned}
$$


$K_{\theta \mathrm{\phi}}$ is the angular stiffness coefficient due to the viscoelastic properties of biarticular muscles spanning the elbow and wrist joints (e.g., FCR, FCU, ECR, ECU). $K_{\theta \theta}$ and $K_{\Phi \Phi}$ are the coefficients due to the sum of the contributions by bi- and monoarticular muscles acting at the elbow (e.g., biceps, triceps) and wrist (e.g., flexor digitorum profundus), respectively (see Mussa-Ivaldi et al., 1985; Lacquaniti and Soechting, 1986). $\Delta \Theta, \Delta \Phi$ are the changes in joint angles from their equilibrium positions (initial conditions), and $\dot{\theta}, \dot{\Phi}$ the rate of such changes. We have made the simplest possible assumptions, namely, that the viscous terms are linear in velocity and are related to the elastic terms via the proportionality constant $C$.

Stiffness coefficients in equation 4 are time-varying. We assume that their time course parallels the changes in muscle tension produced by the observed patterns of EMG activities (Stein and Parmiggiani, 1979). Changes in muscle tension are computed by convolving EMG activity with the muscle twitch profile described by several authors (see Agarwal and Gottlieb, 1982):

$$
\text { Tension }(t)=m \int_{0}^{\infty} u \exp (-20 u) \operatorname{EMG}(t-u) d u
$$

Equations 1, 2, and 3 can be integrated numerically to yield simulated changes in limb kinematics $(\Theta, \dot{\Theta}, \Phi, \dot{\Phi})$. Scaling factors for the 4 stiffncss coefficients $\left(K_{\theta \theta}, K_{\theta \phi}, K_{\phi \Phi}, C\right)$ are then estimated by (least-squares) fitting simulated kinematics to experimental data.

\section{Appendix 2}

\section{Hand stiffness}

For small perturbations about an equilibrium position, the force vector $F$ acting on the limb end point (hand) is related to the linear differential displacements via the end point stiffness matrix $R$ (see Hogan, 1985):

$$
\underline{\mathrm{F}}=\left[\begin{array}{l}
F_{x} \\
F_{y}
\end{array}\right]=-\left[\begin{array}{ll}
R_{x x} & R_{x y} \\
R_{x y} & R_{y y}
\end{array}\right]\left[\begin{array}{l}
d x \\
d y
\end{array}\right]
$$

Similarly, a torque vector $T$ acting on elbow and wrist joints is related to the angular differential displacements via the joint stiffness matrix $\mathbf{K}$ (see Appendix 1):

$$
\underline{\mathbf{T}}=-\left[\begin{array}{cc}
K_{\theta \Theta} & K_{\theta \phi} \\
K_{\theta \Phi} & K_{\Phi \Phi}
\end{array}\right]\left[\begin{array}{l}
d \Theta \\
d \Phi
\end{array}\right]
$$

We want to find a functional relationship between $\mathbf{R}$ and $\mathbf{K}$.

Cartesian coordinates of the end point are related to joint coordinates via

$$
\begin{aligned}
& x=a \cos \Theta+b \cos (\Theta+\Phi), \\
& y=a \sin \Theta+b \sin (\Theta+\Phi),
\end{aligned}
$$

where $a$ and $b$ are the length of the forearm and third metacarpus, respectively (see Fig. 1). The corresponding differential relation is

$$
\left[\begin{array}{l}
d x \\
d y
\end{array}\right]=\mathbf{J}\left[\begin{array}{l}
d \Theta \\
d \Phi
\end{array}\right]
$$

where $\mathbf{J}$ is the Jacobian matrix of transformation:

$$
\mathbf{J}=\left[\begin{array}{cr}
-a \sin \Theta-b \sin (\Theta+\Phi) & -b \sin (\Theta+\Phi) \\
a \cos \Theta+b \cos (\Theta+\Phi) & b \cos (\Theta+\Phi)
\end{array}\right]
$$

If $\mathbf{J}$ is nonsingular, it can be inverted:

$$
\left[\begin{array}{l}
d \Theta \\
d \Phi
\end{array}\right]=\mathbf{J}^{-1}\left[\begin{array}{l}
d x \\
d y
\end{array}\right]
$$

From the principle of virtual work (Goldstein, 1980), we have

$$
\underline{\mathrm{F}}^{T}\left[\begin{array}{l}
\delta x \\
\delta y
\end{array}\right]=\underline{\mathrm{T}}^{T}\left[\begin{array}{l}
\delta \Theta \\
\delta \Phi
\end{array}\right]
$$

where $\underline{F}^{T}$ and $\underline{T}^{T}$ are the transpose of $\underline{F}$ and $\underline{T}$, respectively. Using equations $12,11,6$, and 7 we obtain the desired relationship:

$$
\mathbf{R}=\left[\mathbf{J}^{-1}\right]^{T} \mathbf{K} \mathbf{J}^{-1} \text {. }
$$

In case of a virtual infinitesimal displacement along the vertical ( $\delta x$ $=0$ ), equation 6 gives

$$
\begin{aligned}
& F_{x}=-R_{x y} \delta y \\
& F_{y}=-R_{y y} \delta y
\end{aligned}
$$

\section{References}

Abbs, J. H., and V. L. Gracco (1984) Control of complex motor gestures: Orofacial muscle responses to load perturbations of lip during speech. J. Neurophysiol. 51: 705-723.

Agarwal, G. C., and G. L. Gottlieb (1982) Mathematical modeling and simulation of the postural control loop. Part I. CRC Crit. Rev. Biomed. Engin. 8: 93-134.

Agarwal, G. C., and G. L. Gottlieb (1984) Mathematical modeling and simulation of the postural control loop. Part III. CRC Crit. Rev. Biomed. Engin. 12: 49-93.

Akazawa, K., T. E. Milner, and R. B. Stein (1983) Modulation of reflex EMG and stiffness in response to stretch of human finger muscle. J. Neurophysiol. 49: 16-27.

Alderson, G. J. K., D. J. Sully, and H. G. Sully (1974) An operational analysis of a one-handed catching task using high speed photography. J. Mot. Behav. 6: 217-226.

Andrews, C. J., D. Burke, and J. W. Lance (1972) The response to muscle stretch and shortening in Parkinsonian rigidity. Brain 95: 795812.

Baldissera, F., H. Hultborn, and M. Illert (1981) Integration in spinal neuronal systems. In Handbook of Physiology, Sect. 1, Vol. 2, Part 1, J. M. Brookhart and V. B. Mountcastle, eds., pp. 509-595, American Physiological Society, Bethesda, MD.

Brown, S. H., and J. D. Cooke (1984) Initial agonist burst duration depends on movement amplitude. Exp. Brain Res. 55: 523-527.

Burke, D., S. C. Gandevia, and B. McKeon (1983) The afferent volleys responsible for spinal proprioceptive reflexes in man. J. Physiol. (Lond.) 339: 535-552.

Cole, K. J., and J. H. Abbs (1987) Kinematic and electromyographic responses to perturbation of a rapid grasp. J. Neurophysiol. 57: 14981510.

Delwaide, P. J., P. Crenna, and M. H. Fleron (1981) Cutaneous nerve stimulation and motoneuronal excitability: I. Soleus and tibialis anterior excitability after ipsilateral and contralateral sural nerve stimulation. J. Neurol. Neurosurg. Psychiatry 44: 699-707.

Dietz, V., and J. Noth (1978) Pre-innervation and stretch responses of triceps brachii in man falling with and without visual control. Brain Res. 142: 576-579.

Dufresne, J. R., J. F. Soechting, and C. Terzuolo (1979) Reflex motor output to torque pulses in man: Identification of short- and longlatency loops with individual feedback parameters. Neuroscience 4: 1493-1500.

Ebner, T. J., J. R. Bloedel, J. L. Vitek, and A. B. Schwartz (1982) The effects of cerebellar stimulation on the stretch reflex in the spastic monkey. Brain 105: 425-442.

Eccles, J. C., R. M. Eccles, and A. Lundberg (1957) The convergence of monosynaptic excitatory afferents onto many different species of alpha motoneurones. J. Physiol. (Lond.) 137: 22-50.

Evarts, E. V., U. Shinoda, and S. Wise (1984) Neurophysiological Approaches to Higher Brain Functions, Wiley, New York.

Freund, H. J., and H. J. Büdingen (1978) The relationship between speed and amplitude of the fastest voluntary contractions of human arm muscles. Exp. Brain Res. 31: 1-12.

Georgopoulos, A. P., and J. T. Massey (1988) Cognitive spatial-motor processes. II. Information transmitted by the direction of two-dimensional arm movements and by neuronal populations in primate motor cortex and area 5. Exp. Brain Res. 69: 315-326.

Georgopoulos, A. P., J. F. Kalaska, and J. T. Massey (1981) Spatial trajectories and reaction times of aimed movements: Effects of practice, uncertainty and change in target location. J. Neurophysiol. 46 : $725-743$.

Gibson, J. J. (1966) The Senses Considered as Perceptual Systems, Allen and Unwin, London.

Goldstein, H. (1980) Classical Mechanics, Addison-Wesley, Reading, MA.

Gordon, J., and C. Ghez (1987) Trajectory control in targeted force impulses: II. Pulse height control. Exp. Brain Res. 67: 241-252.

Gottlieb, G. L., and G. C. Agarwal (1980) Response to sudden torques 
about ankle in man. III. Suppression of stretch-evoked responses during phasic contraction. J. Neurophysiol. 44: 233-246.

Gottlieb, G. L., B. M. Myklebust, R. D. Penn, and G. C. Agarwal (1982) Reciprocal excitation of muscle antagonists by the primary afferent pathway. Exp. Brain Res. 46: 454-456.

Grillner, S. (1981) Control of locomotion in bipeds, tetrapods, and fish. In Handbook of Physiology, Sect. 1, Vol. 2, Part 1, J. M. Brookhart and V. B. Mountcastle, eds., pp. 1179-1236, American Physiological Society, Bethesda, MD.

Gurfinkel, V. S., and E. I. Paltsev (1973) Reflex response of antagonist muscles during elicitation of the tendon reflex. Neurophysiology 5: 57-62.

Hammond, P. H., P. A. Merton, and G. G. Sutton (1956) Nervous gradation of muscular contraction. Br. Med. Bull. 12: 214-218.

Hogan, N. (1985) The mechanics of multi-joint posture and movement control. Biol. Cybernet. 52: 315-332.

Houk, J. C., and W. Z. Rymer (1981) Neural control of muscle length and tension. In Handbook of Physiology, Sect. 1, Vol. 1, Part 1, J. M. Brookhart and V. B. Mountcastle, eds., pp. 257-324, American Physiological Society, Bethesda, MD.

Humphrey, D. R., and D. J. Reed (1983) Separate cortical systems for control of joint movement and joint stiffness: Reciprocal activation and coactivation of antagonist muscles. In Motor Control Mechanisms in Health and Disease, J. E. Desmedt, ed., pp. 347-372, Raven, New York.

Jankowska, E., D. McCrea, and R. Mackel (1981) Oligosynaptic excitation of motoneurones by impulses in group la muscle spindle afferents in the cat. J. Physiol. (Lond.) 316:411-425.

Johansson, R. S., and G. Westling (1988) Programmed and triggered actions to rapid load changes during precision grip. Exp. Brain Res. 71: $72-86$.

Karst, G. M., and Z. Hasan (1987) Antagonist muscle activity during human forearm movements under varying kinematic and loading conditions. Exp. Brain Res., 67: 391-401.

Kendall, M. G., and A. Stuart (1969) The Advanced Theory of Statistics, Vols. 1-3, Griffin, London.

Kornblum, S., and J. Requin, eds. (1984) Preparatory States and Processes, Erlbaum, Hillsdale, NJ.

Lacquaniti, F., and C. Maioli (1987) Anticipatory and reflex coactivation of antagonist muscles in catching. Brain Res. 406: 373-378.

Lacquaniti, F., and C. Maioli (1989) Adaptation to suppression of visual information during catching. J. Neurosci. 9: 149-159.

Lacquaniti, F., and J. F. Soechting (1984) Behavior of the stretch reflex in a multi-jointed limb. Brain Res. 311: 161-166.

Lacquaniti, F., and J. F. Soechting (1986) Simulation studies on the control of posture and movement in a multi-jointed limb. Biol. Cybernet. 54: 367-378.

Lacquaniti, F., F. Licata, and J. F. Soechting (1982) The mechanical behavior of the human forearm in response to transient perturbations. Biol. Cybernet. 44: 35-46.

Lakie, M., E. G. Walsh, and G. W. Wright (1984) Resonance at the wrist demonstrated by the use of a torque motor: An instrumental analysis of muscle tone in man. J. Physiol. (Lond.) 353: 265-285.

Lee, D. N. (1980) Visuo-motor coordination in space-time. In Tu- torials in Motor Behavior, G. E. Stelmach and J. Requin, eds., pp. 281-295, North-Holland, Amsterdam.

Matthews, P. B. C. (1972) Mammalian Muscle Receptors and their Central Actions, Arnold, London.

McKinley, P. A., and J. L. Smith (1983) Visual and vestibular contributions to prelanding EMG during jump-down in cats. Exp. Brain Res. 52: 439-448.

Melvill Jones, G., and D. G. D. Watt (1971) Observations on the control of stepping and hopping movements in man. J. Physiol. (Lond.) 219: 709-727.

Mussa-Ivaldi, F. A., N. Hogan, and E. Bizzi (1985) Neural, mechanical and geometric factors subserving arm posture in humans. J. Neurosci. 5: 2732-2743.

Nashner, L. M. (1977) Fixed patterns of rapid postural responses among muscles during stance. Exp. Brain Res. 30:13-24.

Rabiner, L. R., and B. Gold (1975) Theory and Application of Digital Signal Processing, Prentice-Hall, Englewood Cliffs, NJ.

Ross, H. E., E. E. Brodie, and A. J. Benson (1986) Mass-discrimination in weightlessness and readaptation to earth's gravity. Exp. Brain Res. 64: 358-366.

Schomburg, E. D., and H. B. Behrends (1978) The possibility of phasedependent monosynaptic and polysynaptic Ia excitation to homonymous motoneurones during fictive locomotion. Brain Res. 143:533537.

Sharp, R. H., and H. T. A. Whiting (1975) Information-processing and eye movement behavior in a ball catching skill. J. Hum. Mov. Stud. $1:$ 124-131.

Soechting, J. F., and F. Lacquaniti (1981) Invariant characteristics of a pointing movement in man. J. Neurosci. $1: 710-720$.

Soechting, J. F., and F. Lacquaniti (1983) Modification of trajectory of a pointing movement in response to a change in target location. $\mathrm{J}$. Neurophysiol. 49: 548-564.

Soechting, J. F., J. R. Dufresne, and F. Lacquaniti (1981) Time-varying properties of myotatic response in man during some simple motor tasks. J. Neurophysiol. 46: 1226-1243

Stein, R. B., and F. Parmiggiani (1979) The stiffness of slow-twitch muscle lags behind twitch tension: Implications for contractile mechanisms and behavior. Can. J. Physiol. Pharmacol. 57: 1189-1192.

Stevens, S. S. (1975) Psychophysics, Wiley, New York.

Traub, M. M., J. C. Rothwell, and C. D. Marsden (1980) A grab reflex in the human hand. Brain 103: 869-884.

Ullman, S. (1986) Artificial intelligence and the brain: Computational studies of the visual system. Annu. Rev. Neurosci. 9: 1-26.

Watt, D. G. D., E. K. Stauffer, A. Taylor, R. M. Reinking, and D. G. Stuart (1976) Analysis of muscle receptor connections by spiketriggered averaging. I. Spindle primary and tendon organ afferents. J. Neurophysiol. 39: 1375-1395.

Willis, W. D., G. W. Tate, R. D. Ashworth, and J. C. Willis (1966) Monosynaptic excitation of motoneurones of individual forelimb muscles. J. Neurophysiol. 29: 410-424.

Zahalak, G. I., and S. J. Heyman (1979) A quantitative evaluation of the frequency-response characteristics of active human skeletal muscle in vivo. J. Biomech. Engin. 101: 28-37. 